Article publié par le Laboratoire de Construction en Béton de l'EPFL

Paper published by the Structural Concrete Laboratory of EPFL

\begin{tabular}{|l|l|}
\hline Title: & $\begin{array}{l}\text { Review of fundamental assumptions of the Two-Phase Model for aggregate } \\
\text { interlocking in cracked concrete using numerical methods and experimental } \\
\text { evidence }\end{array}$ \\
\hline Authors: & Pundir M., Tirassa M., Fernández Ruiz M., Muttoni A., Anciaux G. \\
\hline Published in: & Cement and Concrete Research, Elsevier \\
\hline $\begin{array}{l}\text { Volume: } \\
\text { Pages: }\end{array}$ & $\begin{array}{l}125(105855) \\
1-17\end{array}$ \\
\hline Year of publication: & 2019 \\
\hline Type of publication: & Peer reviewed journal article \\
\hline
\end{tabular}

\begin{tabular}{|l|l|}
\hline Please quote as: & $\begin{array}{l}\text { Pundir M., Tirassa M., Fernández Ruiz M., Muttoni A., Anciaux G., Review of } \\
\text { fundamental assumptions of the Two-Phase Model for aggregate interlocking in } \\
\text { cracked concrete using numerical methods and experimental evidence, Cement } \\
\text { and Concrete Research, Elsevier, 125(105855), 2019, 1-17. }\end{array}$ \\
\hline
\end{tabular}




\title{
Review of fundamental assumptions of the Two-Phase model for aggregate interlocking in cracked concrete using numerical methods and experimental evidence
}

\author{
Mohit Pundir $^{\mathrm{a}, *}$, Max Tirassa ${ }^{\mathrm{b}}$, Miguel Fernández Ruiz ${ }^{\mathrm{b}}$, Aurelio Muttoni ${ }^{\mathrm{b}}$, Guillaume Anciaux ${ }^{\mathrm{a}}$ \\ a Computational Solid Mechanics Laboratory, ENAC, École Polytechnique Fédérale de Lausanne, Lausanne CH-1015, Switzerland \\ ${ }^{\mathrm{b}}$ Structural Concrete Laboratory, ENAC, École Polytechnique Fédérale de Lausanne, Lausanne CH-1015, Switzerland
}

\section{A R T I C L E I N F O}

\section{Keywords:}

Concrete

Cracks

Shear transfer

Mechanical modelling

Aggregate interlocking

Two-Phase model

\begin{abstract}
A B S T R A C T
Aggregate interlocking allows transferring shear and normal stresses through open cracks, and is considered to significantly contribute to the force transfer in cracked concrete. The complex phenomenon depends on the roughness of cracked surfaces, where material protruding from one side may engage with the opposite one. TwoPhase models were established in the 1980s by Walraven to estimate the force transfer, distinguishing between cement matrix and spherical aggregates. The approach leads to good results but has several shortcomings. In this paper, the fundamental assumptions are reviewed using specific numerical and experimental investigations. Special tests respecting the geometrical assumptions are presented and the results compared with numerically calculated estimates. The model is extended to address some shortcomings and investigate the physical nature of the main parameters. Positive aspects of Two-Phase models and a number of limitations are highlighted, allowing a consistent step forward in the understanding of aggregate interlocking.
\end{abstract}

\section{Introduction}

Concrete is characterised in tension by a low resistance and a small deformation capacity. Unless specific measures are adopted (such as pre-stressing to compensate external actions or providing joints to limit the imposed deformations due to structural and thermal effects), concrete is generally cracked both at serviceability and ultimate limit states. To provide the necessary resistance, reinforcement can be arranged in regions where tension occurs for static reasons (e.g. in the tension side of a bending beam), but the transfer of compression and shear stresses is performed in many cases through cracked regions, which developed due to previous actions. This is for instance shown in Fig. 1, where cracks with a flexural origin (and controlled by the tensile reinforcement) progress and eventually develop inside the location of the theoretical compression strut carrying shear.

The ability of transferring shear and compression stresses through cracks in concrete is governed by the crack opening and sliding as well as the surface properties of the concrete (surface roughness, aggregate and matrix strengths). As shown in the example of Fig. 1b, the kinematics of the lips of the crack generally imply both opening (Mode I kinematics, Fig. 1c) and sliding (Mode II kinematics, Fig. 1d) components. Hence, the kinematic angle $\alpha$ depends on the relative position of the center of rotation which is located approximately at the tip of the shear crack [1-3]. Such general kinematics (Fig. 1e) are usually referred to as Mixed Mode kinematics. Also, it is interesting to note that different kinematics normally develop along a crack and this yields potentially to different capacities to transfer shear and normal forces. The resulting contact forces at the cracked surface (engagement of aggregates and rough surfaces) as a function of the acting kinematics, is usually defined as the aggregate interlocking capacity [4-7]. Investigated in an intensive manner since the 1960s, a number of mechanical approaches have been developed, particularly during the 1980s [6-10] but also more recently $[11,12]$. These approaches gave a comprehensive outlook of the phenomenon and allowed to calculate the interface forces based on the mechanical parameters of concrete. In general, they can be classified depending on the approach followed to model the continuum, which allows to distinguish two general strategies.

The first, referred to as the Homogeneous models, assume that concrete is made of a single homogeneous material, with rough surface profiles taken into account in the shear resistance calculations [7, 11]. The second, referred to as the Two-Phase model, originally proposed by Walraven [6] and with later modifications [13, 14], accounts for heterogeneity of concrete (considering aggregates and mortar), however with a rather simple surface profile. Both approaches predict eventually

\footnotetext{
" Corresponding author.

E-mail address: mohit.pundir@epfl.ch (M. Pundir).
} 


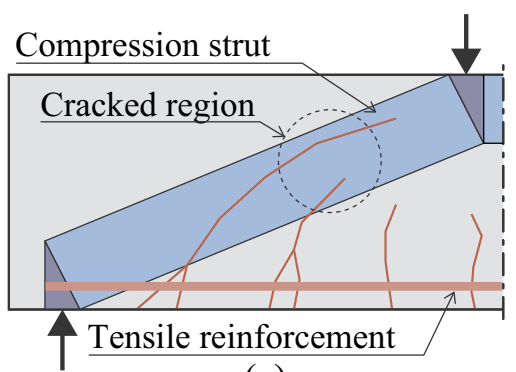

(a)

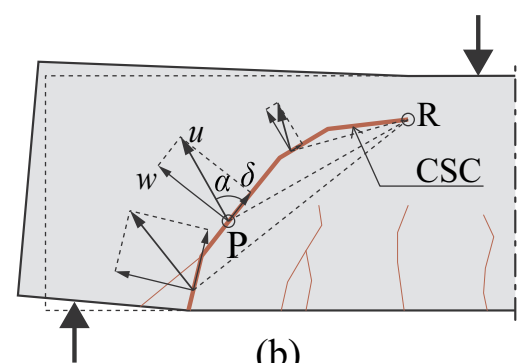

(b)

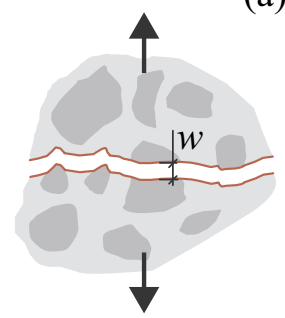

(c)

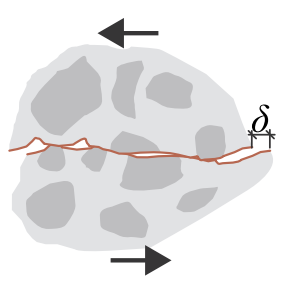

(d)

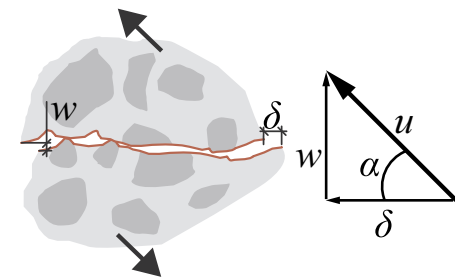

(e)

Fig. 1. (a) Cracks developing through the compression strut in a concrete beam without shear reinforcement (b) Crack pattern before shear failure (c) Mode I kinematics; (d) Mode II kinematics (e) Mixed Mode kinematics.

Table 1

Symbols and notations.

\begin{tabular}{|c|c|c|}
\hline Symbol & Description & $\begin{array}{l}\text { Physical } \\
\text { dimension }\end{array}$ \\
\hline$a$ & Contact area for a single patch & Area \\
\hline$\theta$ & Inclination angle & - \\
\hline$N$ & Number of contact patches & - \\
\hline$A$ & Total contact area & Area \\
\hline$A_{s p}$ & Horizontal projection of all spheres & Area \\
\hline $\boldsymbol{X}$ & Material point position vector & - \\
\hline$\left\{\boldsymbol{e}_{x}, \boldsymbol{e}_{y}, \boldsymbol{e}_{z}\right\}$ & Orthonormal basis & - \\
\hline$\tau$ & Contact stress field & Force/area \\
\hline$F_{N}$ & Total normal force & Force \\
\hline$F_{T}$ & Total shear resistance force & Force \\
\hline$H$ & Hurst exponent & - \\
\hline $\boldsymbol{F}_{R}$ & Total resistance force & Force \\
\hline$\sigma_{p u}$ & Plastic stress threshold & Force/area \\
\hline$\mu$ & Friction coefficient & - \\
\hline $\mathscr{A}$ & Physical space where interlock occurs & Length $^{d-1}$ \\
\hline$u$ & Crack opening & Length \\
\hline$w$ & Crack opening along crack plane & Length \\
\hline$\delta$ & Crack sliding along perpendicular to crack plane & Length \\
\hline$\rho^{N}(a, \theta)$ & $\begin{array}{l}\text { Joined probability density function of contact } \\
\text { patch of area } a \text { and an inclination angle } \theta\end{array}$ & - \\
\hline$\varphi(N)$ & $\begin{array}{l}\text { Probability density function of number of contact } \\
\text { patches }\end{array}$ & - \\
\hline$\Phi(a, \theta, N)$ & Joined probability density & - \\
\hline$\alpha$ & Crack opening angle respective to crack plane & - \\
\hline$D_{\text {steel }}$ & Diameter of steel spheres & Length \\
\hline$D_{\max }$ & Maximum aggregate size in concrete mix & Length \\
\hline$f_{c}$ & Cylinder compressive strength of concrete & Force/area \\
\hline$f_{c m, 28}$ & Average cylinder compressive strength at 28 days & Force/area \\
\hline$f_{c, \text { Test Day }}$ & Cylinder compressive resistance on day of test & Force/area \\
\hline$f_{c, \text { ref }}$ & Reference compressive strength (30 MPa) & Force/area \\
\hline$\eta_{f_{c}}$ & Brittleness factor & - \\
\hline$f_{c p}$ & Equivalent plastic resistance & Force/area \\
\hline
\end{tabular}

the resisting force $\boldsymbol{F}_{R}$ as the following ensemble average,

$\left\langle F_{R}\right\rangle=\iint \phi(\mathscr{A}, N) \cdot N F(\mathscr{A}) d \mathscr{A} d N$

where $\Phi(\mathscr{A}, N)$ is the joined probability density of having one contact patch $\mathscr{A}$, such a contact patch being among $N$ other patches and $\boldsymbol{F}(\mathscr{A})$ is the force produced by such a contact patch. This equation, which will be re-derived in Section 2, is central to Two-Phase models aiming at predicting shear stress/force resistance of a concrete crack. While $\Phi$ describes the stochastic part coming from the random distribution of aggregates, the force $\boldsymbol{F}(\mathscr{A})$ is usually computed with a purely geometrical treatment [6] (Table 1).

In this paper, we will focus only on the Two-Phase models as originally proposed by Walraven [6] and used in many works to model shear [3] or punching cases [14, 15]. Additional hypotheses are introduced in their works in order to find semi-analytical forms of the probability density $(\Phi)$ and the transmitted force $(\boldsymbol{F})$. These hypotheses can be divided into two categories: geometrical and material. The geometrical hypotheses state that the crack surface profile is planar with spherical aggregates protruding partially from it. Fig. 2 presents such an approach. The probability density $\Phi$ can be derived as a function of both the packing density of the concrete and of the distribution of aggregate sizes (through a grading curve as in Ref. [16]). In Ref. [6], the material hypotheses state that the deformation is undertaken mostly by the matrix, so that it is idealized as perfectly-plastic whereas aggregates are considered as rigid. Lastly, in the original formulation of Walraven, the surface profile is un-physically assumed to remain unchanged after being in contact. There are two material freeparameters for this model: a plastic stress threshold $\sigma_{p u}$, and a friction coefficient $\mu$.

This approach generally provides reasonable estimates of the maximum normal and tangential stresses [17], but is less accurate in the post-peak phase where it tends to overestimate the transferred forces [3]. Moreover, the assumption of perfectly spherical and rigid aggregates may be very unrealistic depending on the type of aggregates effectively used. Finally, the physical nature of the free-parameters $\sigma_{p u}$ and $\mu$ remains unclear, as they are difficult to measure directly and were likely adapted to better fit experimental results.

The aim of this paper is to better understand the consistency of the Two-Phase model approach and particularly of the physical interpretation of the parameters $\sigma_{p u}$ and $\mu$. An extension of the model will be suggested by introducing the induced surface alterations, which will allow accurate semi-analytic predictions of shear stress resistance. This new model will be strengthened by a number of tailored experiments, comprising concrete with various aggregate sizes, and various loading kinematics. Furthermore, the new model being an extension of Walraven's model uses only a few material parameters $\left(\sigma_{p u}, \mu\right)$ as well as parameters $\left(\beta_{y}, \beta_{z}\right)$ to predict the complex mechanical mechanism (elasto-plastic deformation, degradation of matrix material) at the crack interface. Being simple and purely geometric in nature, the model does 


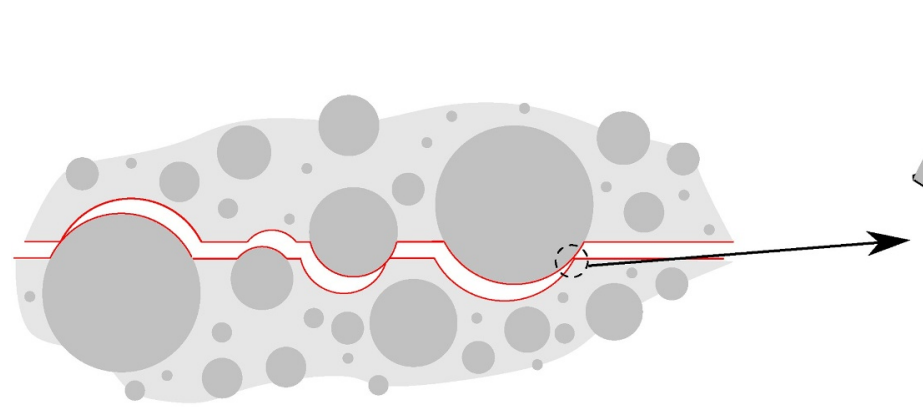

(a)

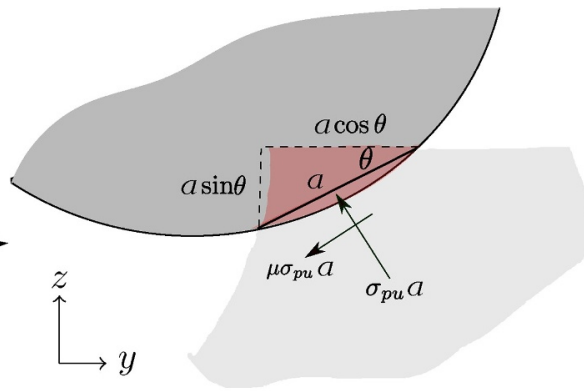

(b)

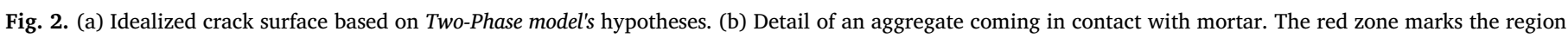
where mortar is being plasticized. (For interpretation of the references to color in this figure legend, the reader is referred to the web version of this article.)

possesses some limitations which are discussed in detail in later sections.

This paper starts in Section 2 by reviewing and reformulating the Two-Phase problem, which will describe the necessary mathematical concepts. Section 3 describes the experimental setup, which is designed to remain as close as possible to the hypotheses of the presented models. In Section 4.1, the Two-Phase model is extended to predict shear stress resistance for a 3D geometry. New predictions made by this model will be compared with experimental results, which allow to highlight the situations where the original model does not work accurately. Then, a new semi-analytical model acknowledging the surface alterations due to contact and the elasto-plastic behaviour of the problem is introduced in Sections 4.2 and 4.3. With such an approach, a fine agreement with experiments will be demonstrated. Finally, Section 5 discusses the implications and perspectives brought by this model, especially in view of making predictions for complex crack roughness, therefore involving a statistical treatment.

\section{Two-Phase model}

In this section, Walraven's approach [6] is reviewed, starting with the derivation of it's central Eq. (1). To do so, the resisting force $\boldsymbol{F}_{R}$ is calculated as the sum of all the forces acting along the contact area $A$ on the crack surface, which provides the general definition:

$\boldsymbol{F}_{R}=\int_{A} \tau(\boldsymbol{X}) \boldsymbol{n} d S$

where $\tau$ refers to the contact stress field and $\boldsymbol{n}$ the unit vector field normal to the surface. The contact area $A$ can be decomposed into $N$ smaller contacting patches $\left(a_{i}\right)_{i=1 \ldots N}$ such that $\sum_{i}^{N} a_{i}=A$. The average contact force produced by these patches can be linked to the total force with:

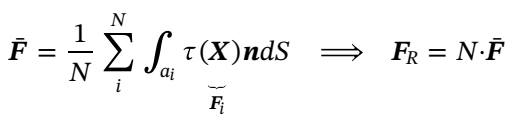

Assuming numerous contact patches and a faithful statistical representation, $\overline{\boldsymbol{F}}$ can be expressed with a probability density integral:

$\overline{\boldsymbol{F}}=\frac{1}{N} \sum_{i}^{N} F_{i}=\int \rho^{N}(\mathscr{A}) \cdot \boldsymbol{F}(\mathscr{A}) d \mathscr{A}$

where $\rho^{N}(\mathscr{A})$ is the joined probability density of having a contact patch $\mathscr{A}$, knowing that there are $N$ patches in total. On the other end, $\boldsymbol{F}(\mathscr{A})$ is the force produced by a contact patch. When the probability density for the number of contact patches $(\varphi)$ is provided, the expectation of the resistance force Eq. (1) can be obtained by combining Eqs. (3) and (4):

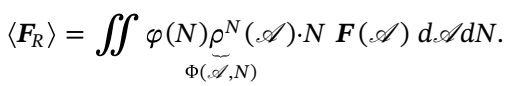

In this equation, $\Phi$ reflects the stochastic nature of surface topologies produced during concrete cracking, as well as the current level of opening and shear displacements. In order to obtain a formal expression, Walraven employed a 2D geometry where the crack surface is nominally flat with perfectly circular protruding aggregates, globally following a specified density and a specified distribution of radii (Fuller curve). Also, the opening and shear displacements were assumed to be homogeneous throughout the entire crack surface. In a 2D setup, any contact patch $\mathscr{A}$ is simply characterised with a contact area $a$ and an inclination angle $\theta$ leading to:

$\left\langle\boldsymbol{F}_{R}\right\rangle=\iiint \Phi(a, \theta, N) \cdot N \boldsymbol{F}(a, \theta) d a d \theta d N$.

Also, thanks to this simple geometry, the resistance force $\boldsymbol{F}(a, \theta)$ can be geometrically constructed. For a given opening displacement $w$ and shear displacement $\delta$, the interlocking situation is as shown in Fig. 2. The overlapping volume between mortar and aggregates (shown in red colour on the Figure) allows to define a contacting plane resulting from plastic deformation, and to associate it with a contact area and an inclination angle $(a, \theta)$. Therefore, the force produced becomes the following line integral

$\boldsymbol{F}(a, \theta)=\int_{0}^{a} \tau(\underbrace{l, \theta}_{\tau^{P}}) \boldsymbol{n} d l$

where the stress $\tau^{P}$ is naturally decomposed into a normal contribution preventing interpenetration and a tangential component resulting from Coulomb friction forces

$\tau^{P}(l, \theta)=p(l)\left[\begin{array}{cc}1 & \mu \\ -\mu & 1\end{array}\right]\left(\begin{array}{l}\sin (\theta) \\ \cos (\theta)\end{array}\right)=p(l)\left(\begin{array}{l}\sin (\theta)+\mu \cdot \cos (\theta) \\ \cos (\theta)-\mu \cdot \sin (\theta)\end{array}\right)$

where $p$ is the normal load field and $\mu$ is the Coulomb friction coefficient. Under a perfectly-plastic assumption, $p$ becomes the constant $\sigma_{p u}$ and we obtain the following expression:

$\boldsymbol{F}(a, \theta)=a \cdot \sigma_{p u}\left(\begin{array}{l}\sin (\theta)+\mu \cdot \cos (\theta) \\ \cos (\theta)-\mu \cdot \sin (\theta)\end{array}\right)$

With the final equation that Walraven produced being

$\left\langle\boldsymbol{F}_{R}\right\rangle=\sigma_{p u} \iiint a N\left(\begin{array}{l}\sin (\theta)+\mu \cdot \cos (\theta) \\ \cos (\theta)-\mu \cdot \sin (\theta)\end{array}\right) \Phi(a, \theta, N) d a d \theta d N$.

Actually, Walraven's equation is written in the form

$\left\langle\boldsymbol{F}_{R}\right\rangle=\iint \Psi(D, h) \boldsymbol{F}(D, h) d D d h$.

where the probability density $\Psi$ and the force $\boldsymbol{F}(D, h)$ are resulting from an integration of the variable $N$ followed by a change of variable $(a, \theta)$ $\rightarrow(D, h)$, where $D$ represents aggregate diameters and $h$ represents the elevation (distance to crack plane) at which aggregates are cut. This comes from the manner Walraven used to derive this expression (by 


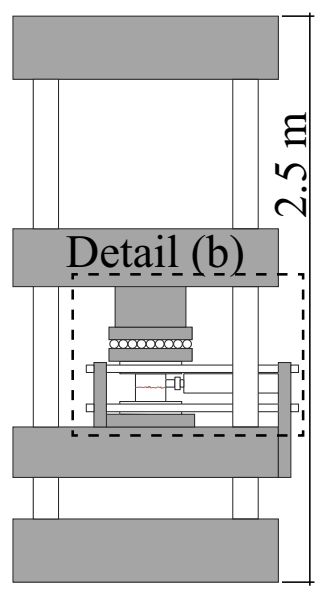

(a)

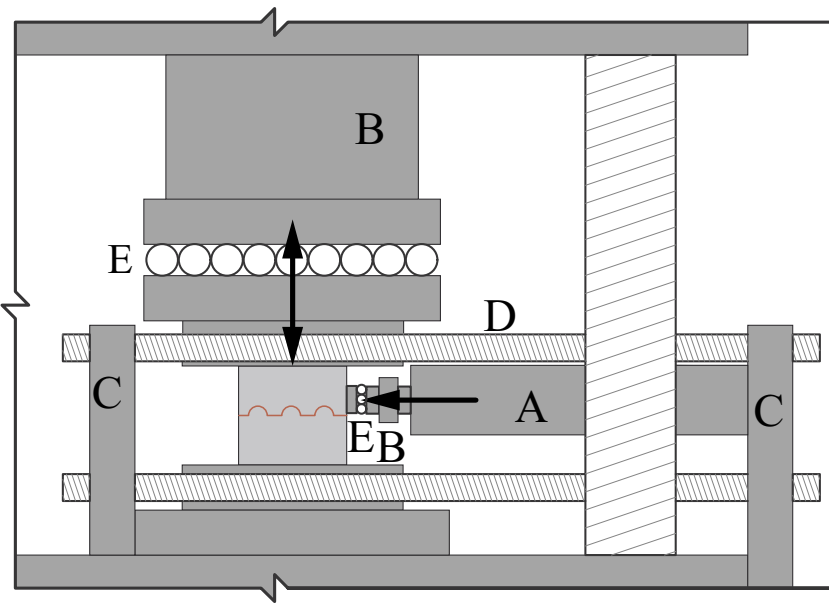

(b)

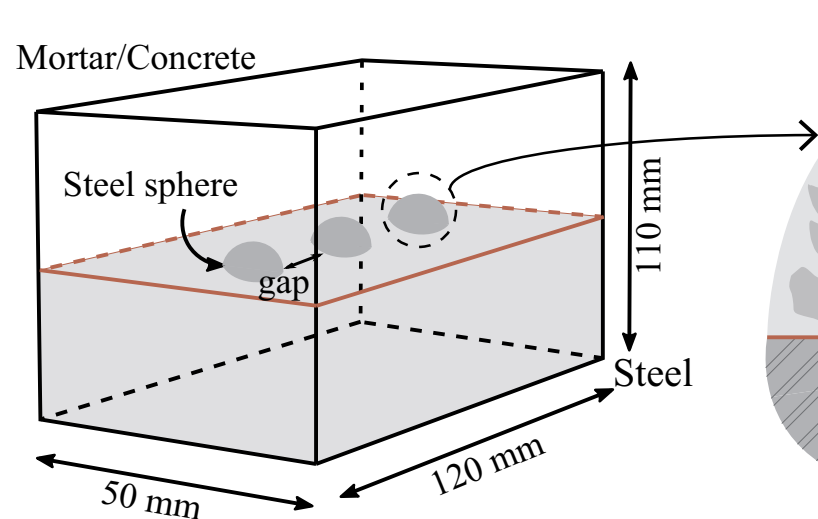

(c)

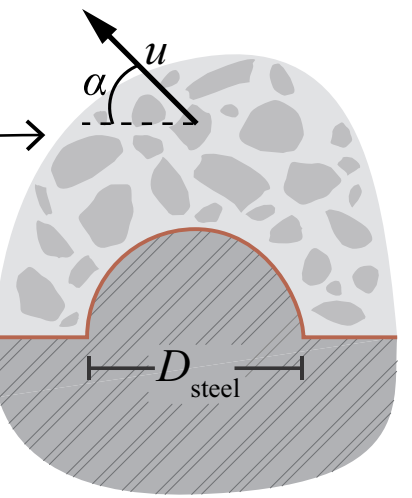

(d)

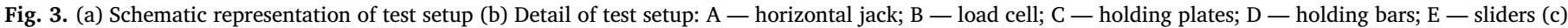
Schematic representation of typical specimen (d) Section through a sphere.

using Fuller's curve and density constraints). Therefore, the probability for the number of contact patches is not appearing, even though it is taken into account to obtain the analytical expressions reported in Ref. [6].

Several remarks can be pointed out on Walraven's model. First, it is only a 2D model with purely circular aggregates, leading to possible errors, in computing $\boldsymbol{F}(D, h)$, coming from the limitations of the geometry description. Second, the actual cracked surfaces have profiles with a roughness generally characterised by a Hurst exponent $H$, which ranges from 0.6 to 0.8 for naturally occurring rough surfaces $[18,19]$. The Hurst exponent $H_{\text {ideal }}$ for the surface profile considered in TwoPhase model is found out to be in the range 1.0-1.1(for further detail refer to Appendix A). This oversimplification of cracked surfaces leads to possible errors in the probability density $\Psi$ which accounts for the number of contact patches. Third, there are only two parameters in the model characterising the matrix (assumed to be homogeneous), namely the plastic stress threshold $\sigma_{p u}$ and the friction coefficient $\mu$. These values are usually determined by fitting the experimental values. Last, the assumed constitutive behaviour is perfectly-plastic.

The aim of the presented work is to analyse in depth the interlock elasto-plastic mechanical response, which is modelled by the function $F$ $(a, \theta)$ in Eqs. (1) and (6). To that end, a 3D geometry made with few spherical aggregates of the same radius will be employed, therefore discarding any stochastic contribution. In the next section, the employed experimental setup will be introduced, immediately followed by the achieved experimental results. A novel predictive model will be presented in latest sections.

\section{Experimental programme}

\subsection{Test-setup}

To validate the Two-Phase model, 23 experiments respecting its two main geometrical hypotheses (globally planar cracks and rigid, spherical aggregates) were carried out. To this purpose, three steel halfspheres of identical diameter were fixed every $30 \mathrm{~mm}$ to one of the sides of a polished steel cuboid. The spherical shape was selected to match the idealized aggregates of the Two-Phase model. To average the material's local variability, three spheres were used for each test. In addition, the distance between spheres (spaced 7 to 10 times the sphere radius) was set to limit the potential interaction between the contact regions, to reproduce again in a close manner the basic assumptions of the Two-Phase model. All steel parts were made with S235JR + C steel, in order to avoid any potential rupture of the sphere (as would potentially occur for lightweight or weak aggregate [20]). The specimens were identical except for the diameters of the three half-spheres $D_{\text {steel, }}$, which measured 6 or $8 \mathrm{~mm}$ depending on the specimen. A formwork was then fixed to the cuboid in order to cast concrete or mortar on top of the surface with the three half-spheres. The final specimens consisted thus of a steel part and a cement-material part and were $120 \mathrm{~mm}$ wide, $110 \mathrm{~mm}$ high and $50 \mathrm{~mm}$ thick, as shown in Fig. 3c. The casting was done using five different mixes with varying the maximum aggregate size $D_{\max }$, as reported in Table 2. Series 3101,3102 and 3103 were made using concrete, while series 3104 and 3105 were produced with a mortar mix similar to the one prescribed by EN 196-1 [21].

Fig. 4 provides some additional information on the aggregates used 
Table 2

Mix designs for the used concrete castings. $f_{c \text {,Test Day }}$ is estimated in accordance to MC 2010 [23] (values marked with an asterisk refer to values from a comparable casting).

\begin{tabular}{clllll}
\hline & \multicolumn{2}{l}{ Series \# } & & & \\
\cline { 2 - 5 } & 3101 & 3102 & 3103 & 3104 & 3105 \\
\hline$D_{\max }[\mathrm{mm}]$ & 8 & 16 & 16 & 2 & 2 \\
$\begin{array}{c}\text { Water }\left[\mathrm{kg} / \mathrm{m}^{3}\right] \\
\text { Cement }[\mathrm{kg} /\end{array}$ & 177 & $195^{*}$ & 165 & 500 & 500 \\
$\left.\mathrm{~m}^{3}\right]$ & 321 & $340^{*}$ & 330 & 1000 & 1000 \\
$\begin{array}{c}\text { Cement type } \\
\text { CEMII }\end{array}$ & CEMII & CEMII & CEMII & CEMII \\
$\begin{array}{c}\text { Aggregates } \\
{\left[\mathrm{kg} / \mathrm{m}^{3}\right]}\end{array}$ & 1853 & $\begin{array}{l}\text { L-LL42.5N } \\
\text { L-M-T- }\end{array}$ & A-LL42.5N & A-LL42.5N & A-LL42.5N \\
$f_{c m, 28}[\mathrm{MPa}]$ & 40.2 & 38.7 & 1880 & 3000 & 3000 \\
$f_{c, \text { Test Day }}[\mathrm{MPa}]$ & 43.7 & 43.0 & 61.1 & 38.3 & 44.3 \\
\hline
\end{tabular}

for the different castings. The gravel used for \#3102 consisted prevalently (>90\%) of limestone classified as medium-hard according to the Swiss code SN670115 [22]. Castings \#3101 and \#3103 contained mainly aggregates made of medium-hard limestone $(\sim 85 \%$, see aggregate curves in Fig. 4 and details in Ref. [17], casting \#06). Finally, ordinary quartz-sand with a granulometry similar to the one of the standard-sand prescribed by EN 196-1 [21] was used for the mortar specimens (see Fig. 4c).

After casting, the specimens were cured under sealed conditions for at least 28 days (typically around 6 weeks) and then de-moulded and prepared for testing. Devices measuring crack opening and sliding across the interface between steel and concrete were fixed on the specimen, which was then glued on the steel plates of a $500 \mathrm{kN}$ electromechanical testing machine, shown in Fig. $3 \mathrm{a}$ and described more extensively in reference [17]. The machine was capable to impose displacements in the vertical direction of the specimen, equivalent to a Mode I opening. Through the addition of a $50 \mathrm{kN}$ hydraulic jack in horizontal position it was also possible to push sideways on the upper half of the specimen, in order to apply Mode II kinematics to the analysed interface. Low-friction linear guides allowed for movement of the upper part of the specimen during testing, and load cells enabled the measurement of the applied vertical and horizontal forces. Using a control unit with a closed-controlled loop it was possible to coordinate the displacements applied in the two directions, and to apply predefined Mixed Mode kinematics on the crack. These kinematics were characterised by a constant opening angle $\alpha$ as shown in Fig. 1e, which was one of the main test parameters in addition to the material properties and the half-sphere diameter $D_{\text {steel. }}$. A summary of the performed tests is given in Table 3 , where the first four digits of the specimen name indicate the concrete or mortar mix.
Table 3

Test specimens, spheres diameter and applied kinematics; the asterisks indicate tests with special kinematics as described in the text.

\begin{tabular}{|c|c|c|c|c|c|}
\hline \multicolumn{3}{|c|}{ Concrete specimens } & \multicolumn{3}{|c|}{ Mortar specimens } \\
\hline$\#$ & $D_{\text {steel }}[\mathrm{mm}]$ & $\alpha\left[^{\circ}\right]$ & \# & $D_{\text {steel }}[\mathrm{mm}]$ & $\alpha\left[^{\circ}\right]$ \\
\hline 310101 & 8 & 30 & 310401 & 6 & 30 \\
\hline 310102 & 6 & 25 & 310402 & 6 & 20 \\
\hline 310103 & 6 & 25 & 310403 & 6 & 15 \\
\hline 310104 & 8 & 25 & 310404 & 8 & 20 \\
\hline 310201 & 6 & 25 & 310405 & 8 & 25 \\
\hline 310202 & 6 & 20 & 310406 & 8 & 30 \\
\hline 310204 & 8 & 25 & 310502 & 6 & 25 \\
\hline 310301 & 8 & 25 & 310503 & 6 & 15 \\
\hline 310302 & 8 & 30 & 310504 & 8 & 15 \\
\hline 310303 & 6 & $0 *$ & 310505 & 8 & 20 \\
\hline 310304 & 6 & 25 & 310506 & 8 & 30 \\
\hline 310305 & 0 & $90 *$ & & & \\
\hline
\end{tabular}

The table includes two specimens tested with special kinematics:

- Specimen 310305 had no spheres crossing the steel/concrete interface and was tested in Mode I to verify if adherence between the two materials was present. The maximum tensile force which was measured was about $20 \mathrm{~N}$. Thus, the adherence between the planar surfaces can be considered as existent but negligible compared to the force measured during the other tests. This result is further confirmed by the Mode I phase of test 310303 which leads to similar results.

- Specimen 310303 was tested following Mode I kinematics until an initial crack opening $w_{\text {init }}$ of $0.5 \mathrm{~mm}$ was reached; then pure Mode II was applied at constant crack opening $\left(\alpha=0^{\circ}\right)$. During this second phase, the measured forces increased until they reached the load limit of the setup and the experiment had to be stopped. At that instant both the vertical and the horizontal load measured about $16 \mathrm{kN}$. After removal of the specimen it could be seen that the high forces had caused the steel spheres to plastically deform at their tips. This shows that, without dilatation, aggregate interlocking forces can reach very high values and the local stresses may reach the plastic steel resistance, invalidating the geometrical assumption of perfect spheres.

\subsection{Tests with concrete specimens}

The 12 concrete specimens were cast using three different mixtures (Table 2, the specimens with mortar will be detailed later): Series 3101 and 3102 had a similar compressive strength but varied in maximum aggregate size, while the concrete of series 3103 had a 50\% higher resistance. Moreover, a red pigment was added to the mix 3101,

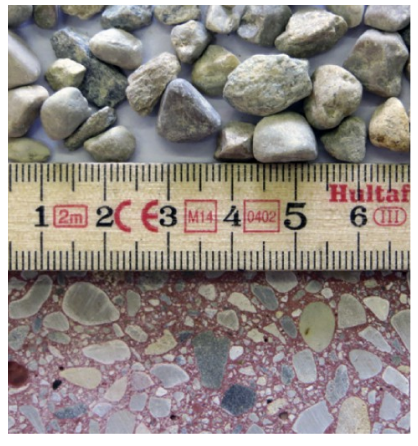

(a)

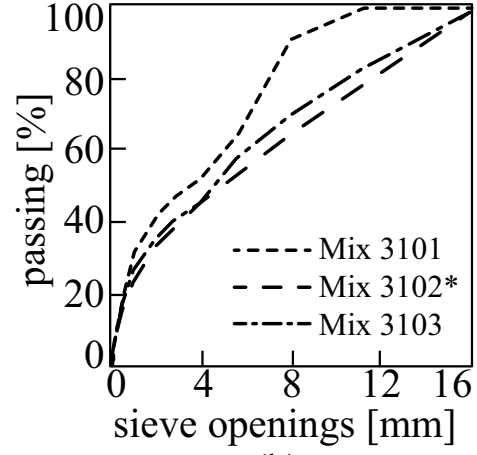

(b)

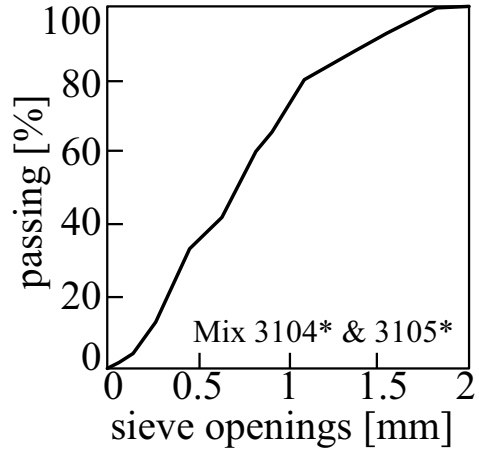

(c)

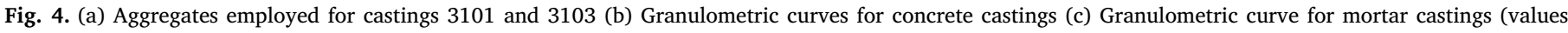
marked with an asterisk refer to values from a comparable casting). 
allowing to better distinguish crushed sand and aggregates (Fig. 8). Fig. 5 reports the measured forces for all Mixed Mode tests, normalised as follows:

$\frac{\tau}{f_{c p}}=\frac{F_{T}}{3 A_{s p} f_{c p}} \quad$ and $\quad \frac{\sigma}{f_{c p}}=\frac{F_{N}}{3 A_{s p} f_{c p}}$,

where $F_{T}$ is the measured tangential force, $F_{N}$ is the measured normal force, $A_{s p}=\pi D_{\text {steel }}{ }^{2} / 4$ is the area of the horizontal projection of a sphere and $f_{c p}$ is the equivalent plastic compressive resistance of the material accounting for the material brittleness in compression and for the fact that the plastification is a gradual process (with regions in the softening phase while others attain the material strength $[24,25]$ ). According to Model Code 2010 [23], this value can be estimated as:

$f_{c p}=\eta_{f_{c}} f_{c}, \quad$ where $\quad \eta_{f_{c}}=\left(\frac{f_{c, \text { ref }}}{f_{c}}\right)^{\frac{1}{3}} \leq 1$

A suitable value for $f_{c, \text { ref }}$ is $30 \mathrm{MPa}[23,25]$. Each test is plotted as four curves in a graph with four axes, to better show the relationship between the measured values of $\tau, \sigma, \delta$ and $w$.

Some curves, like the ones relative to the shear stress of tests 310202, 310304, 310101 and 310104, follow a clear trajectory: they start with a stiff, almost linear ascending phase, followed by a nonlinear phase as they approach the maximum shear load $\tau_{\max }$ and end with a gradual softening phase. Others however do not behave so consistently and present some scatter.

For example, during test 310102 the shear force started to increase again after the first peak load. In other cases, like for test 310301, the maximum shear force is not clearly defined and a large plateau is recorded. Finally, for test 310302 , the shear force decreases very rapidly after the peak load, before it reverts to the typical rate of other tests.

Such randomness can be potentially attributed to the heterogeneous nature of the concrete material: when the steel spheres mostly interact with the cement matrix and small sand particles, the behaviour is clear and consistent. However, when the spheres enter in contact with a large aggregate, a more random behaviour can be observed, depending on the size, shape and hardness of the aggregate. For example, Fig. 8 (e) shows the surface of the aforementioned specimen 310102 after the test. A large aggregate (length $>10 \mathrm{~mm}$ ) has been clearly revealed by one of the spheres scraping off the surrounding material.

With respect to the behaviour of specimen 310204, the results are not considered valid for larger displacements as two parts of the device measuring the crack opening and sliding entered in contact, influencing the force transfer and resulting in the pronounced force plateau. The test is thus marked with an asterisk in Fig. 5.

The values of $\tau$ and $\sigma$ occurring when $\tau=\tau_{\max }$ are marked with an $X$

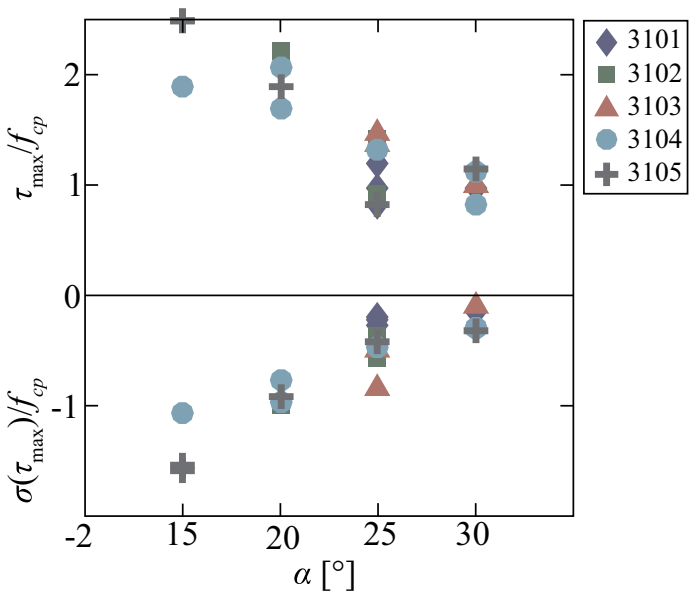

Fig. 6. Normalised stresses occurring at $\tau_{\max }$ as a function of $\alpha$.

in Fig. 5. Note that this does not correspond to the maximum value of $\sigma$, as the normal force usually reaches its peak only after the maximum tangential force is measured. According to the approach of Walraven $(\mu$ is constant) this can only be explained by a change in the angle of the plasticized region with respect to the crack plane (Fig. 2). These peak values are compared to each other in Fig. 6, where they are plotted as a function of the applied angle $\alpha$. The plot shows a clear trend, as the peak forces decrease consistently with increasing Mixed-Mode angle regardless of the various used materials.

\subsection{Test with mortar specimens}

In order to limit the random material response observed for the concrete specimens (direct contact of spheres and aggregates), 11 mortar specimens were additionally tested. The mix-design for the material was prepared according to EN 196-1 [21], using ordinary sand with $D_{\max }=2 \mathrm{~mm}$. Series 3105 had a higher compressive resistance on the test-day compared to series 3104 and typically resulted in higher forces. As shown in Fig. 7, the curves are better defined than the ones for the concrete specimens, generally showing one clearly identified force peak followed by the softening phase. The trends observed for the concrete specimens remain visible. An exception is presented by tests 310403 and 310503 ( $D_{\text {steel }}=6 \mathrm{~mm}, \alpha=15^{\circ}$ ), where the forces remain relatively high even after the peak force. A similar response in terms of $\tau_{\max }$ and $\sigma_{\max }$ was observed as for concrete specimens (see Fig. 7) with a delayed occurrence of $\sigma_{\max }$ with respect to $\tau_{\max }$.

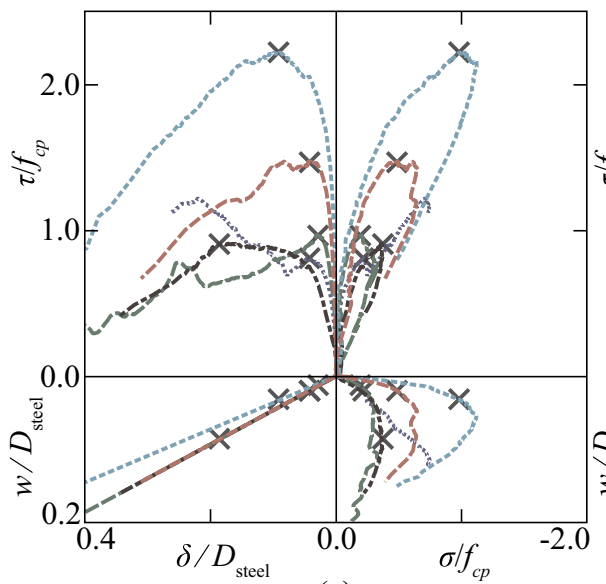

(a)

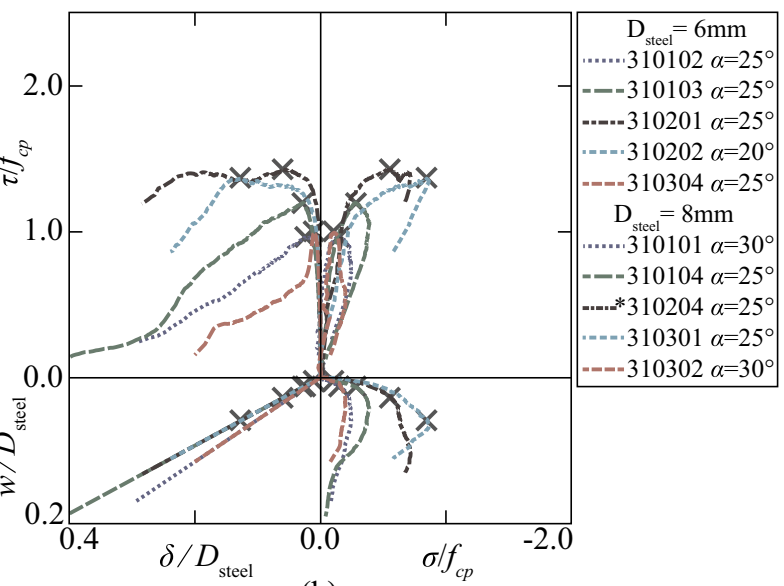

(b)

Fig. 5. Experimental results for interlocking tests between concrete and steel spheres (a) $D_{\text {steel }}=6 \mathrm{~mm}$ (b) $D_{\text {steel }}=8 \mathrm{~mm}$. 


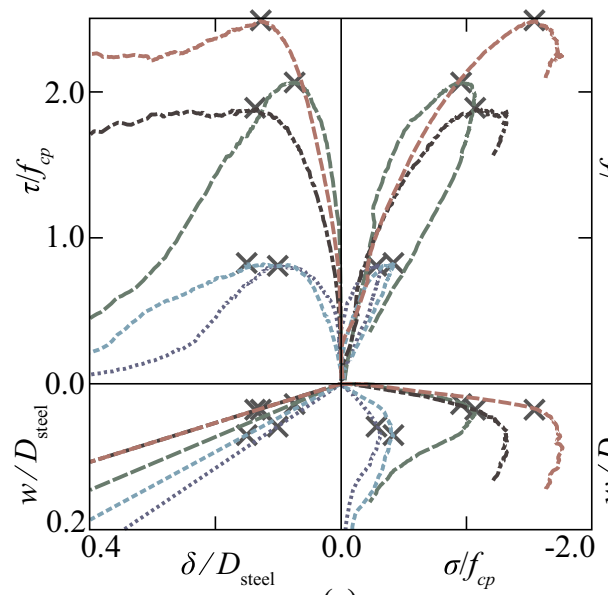

(a)

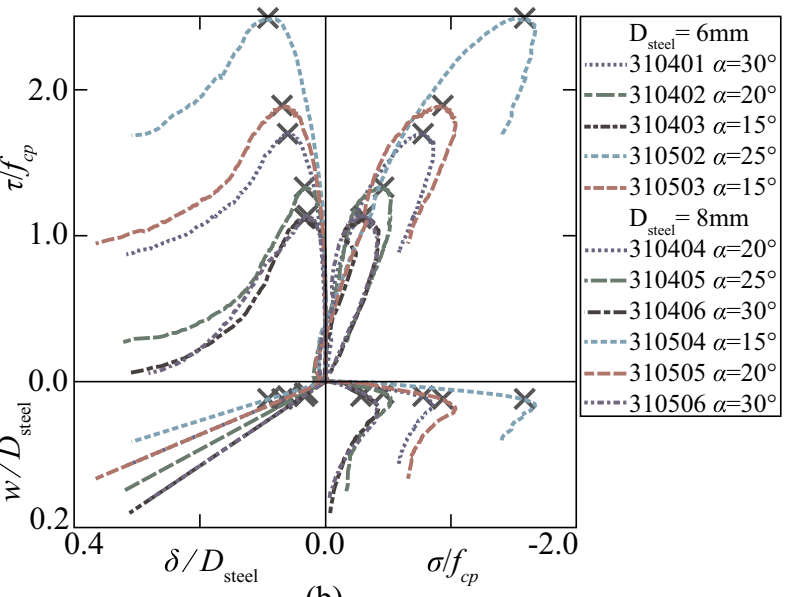

(b)

Fig. 7. Experimental results for interlocking tests between mortar and steel spheres (a) $D_{\text {steel }}=6 \mathrm{~mm}$ (b) $D_{\text {steel }}=8 \mathrm{~mm}$.

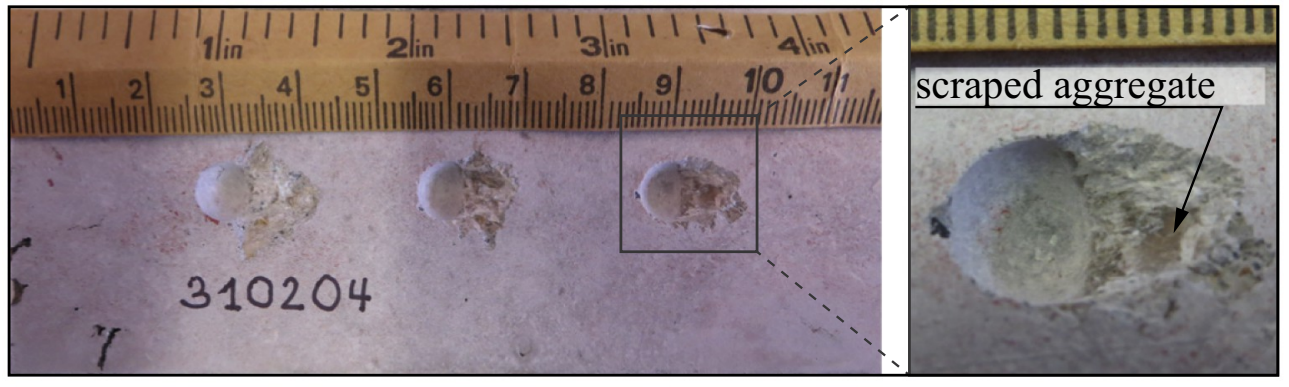

(a)

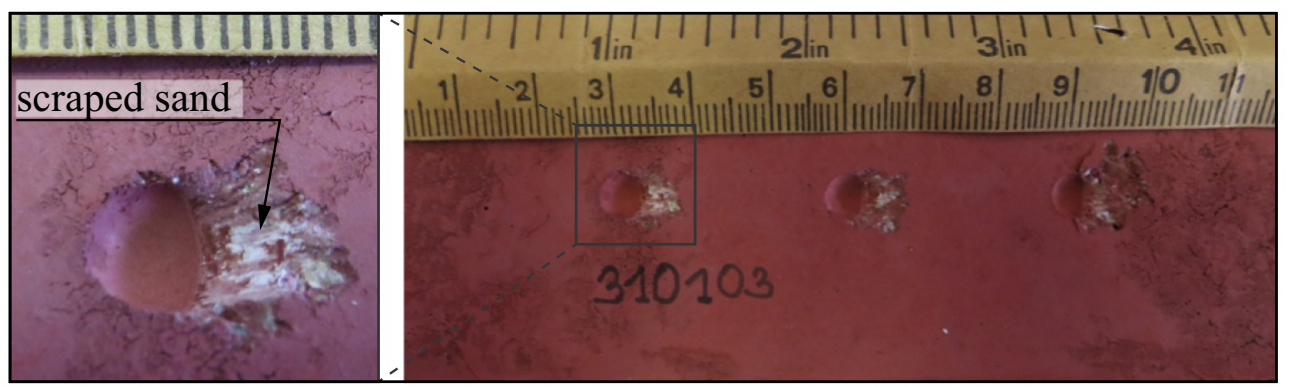

(c)
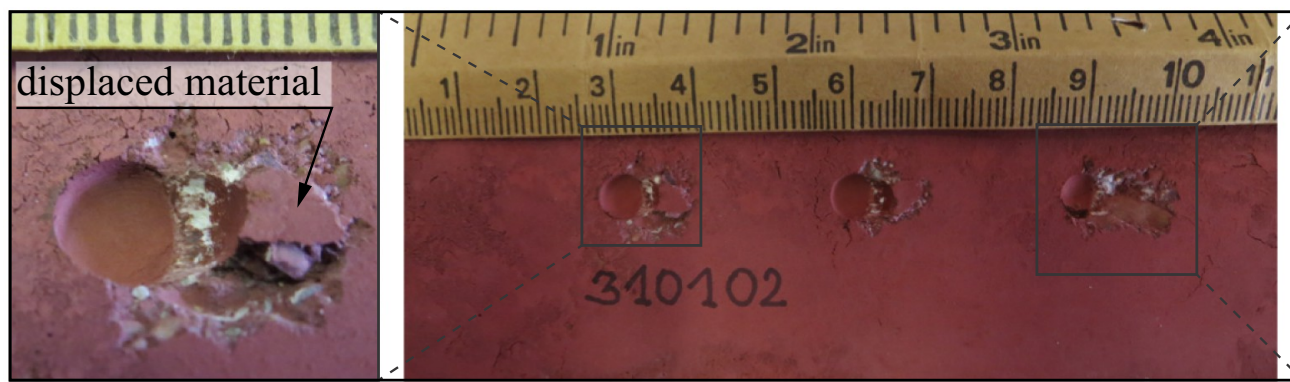

(e)

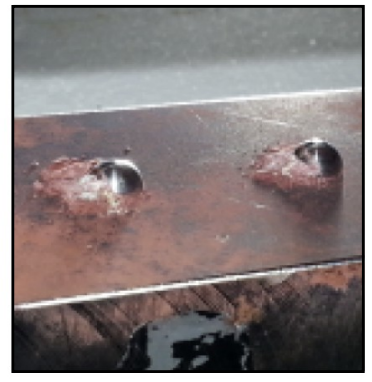

(b)

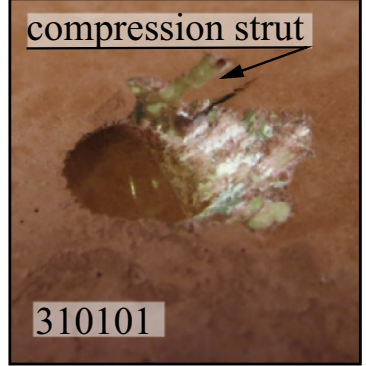

(d)

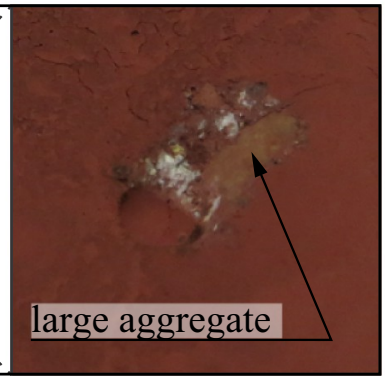

arge aggregate

Fig. 8. Pictures of tested specimens: (a) Concrete surface of specimen 310204 after testing: the void due to the presence of the steel sphere and the damage due to the applied kinematics are visible. A scraped aggregate is visible in the damaged zone; (b) Steel block after testing: crushed concrete material is visible on one side of the spheres; (c)-(d) Concrete surface after testing: A red pigment was added to mix 3101 making the crushed, white material clearly visible; (e) Concrete surface of specimen 310102 after testing: on the left, cohesive material pushed away by one of the spheres; on the right a large aggregate is visible next to one of the voids, possibly explaining the anomalous softening behaviour of this test. (For interpretation of the references to color in this figure legend, the reader is referred to the web version of this article.) 


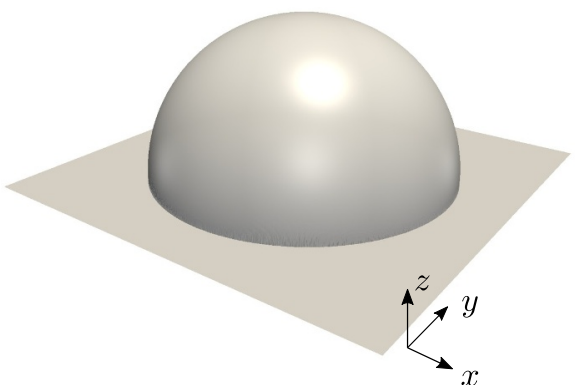

(a)

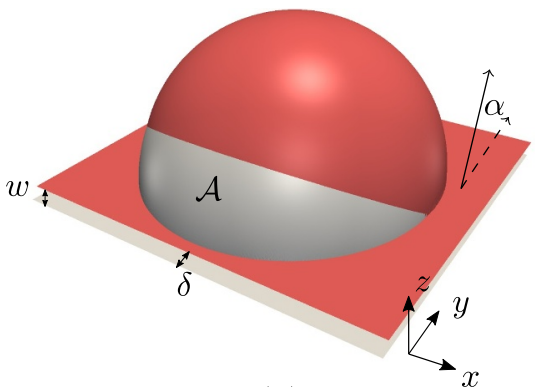

(b)

Fig. 9. (a) Bottom sphere in an interlock-situation. (b) Crack-opening between bottom sphere (gray) and upper sphere (red) for a given shear opening $\delta$ and a vertical opening $w=\delta \tan \alpha$. The interlocking contact patch $\mathscr{A}$ is the remaining gray region, visible because the bottom surface stays above the upper surface in this configuration. (For interpretation of the references to color in this figure legend, the reader is referred to the web version of this article.)

\section{Review and extension of the Two-Phase model}

\subsection{Review of original formulation according to Walraven}

The interpretation of the described tests with steel spheres is made by assuming that the distance between spheres is sufficient to avoid any interaction between them. Therefore, a single sphere will be numerically modelled and a proper normalisation will be used to make the comparison with experiments. Starting from the Two-Phase model governing Eq. (1), the probability density function $\Phi$ is now discrete since randomness was fully removed, therefore leading to the simplified shear resistance equation expressed after projection to the direction $\boldsymbol{e}_{\boldsymbol{y}}$ :

$\left\langle F_{T}\right\rangle=3 F\left(\mathscr{A}\left(w, \delta, D_{\text {steel }}\right)\right)$

where $\mathscr{A}$ is the region of space where contact/interlocking occurs. Similar to experimental results, the total shear resistance force $\left\langle F_{T}\right\rangle$ is normalised by $3 A_{s p} f_{c p}$ where $A_{s p}=\pi D_{\text {steel }}^{2} / 4$ and $f_{c p}$ is the equivalent plastic resistance. In a 2D setup, $\mathscr{A}$ can be represented by the area $a$ and the inclination $\theta$, which is not possible anymore for the more general $3 \mathrm{D}$ case. $\mathscr{A}$ is expressed as a function of the opening vector $(w, \delta)$. The purpose of this section is two-fold. First an expression for $\mathscr{A}\left(w, \delta, D_{\text {steel }}\right)$ is provided in the case of a spherical aggregate. Secondly a perfectlyplastic constitutive behaviour will be used to compare with the experimental results. Few modifications are required to adapt the TwoPhase model to the investigated case. By definition, we have in the perfectly-plastic case:

$$
\begin{aligned}
F(\mathscr{A}) & =\int_{\mathscr{A}} \tau^{\boldsymbol{P}}(\boldsymbol{X}) \cdot \boldsymbol{e}_{\boldsymbol{y}} d S=\sigma_{p u} \int_{\mathscr{A}}(\boldsymbol{n}(\boldsymbol{X})+\mu \boldsymbol{t}(\boldsymbol{X})) \cdot \boldsymbol{e}_{\boldsymbol{y}} d S \\
& =\sigma_{p u} \int_{\mathscr{A}} \underset{\tilde{A}_{y}^{n}}{\boldsymbol{n}(\boldsymbol{X}) \cdot \boldsymbol{e}_{\boldsymbol{y}}} d S+\sigma_{p u} \mu \int_{\mathscr{A}} \underset{\tilde{A}_{y}^{t}}{\boldsymbol{t}(\boldsymbol{X}) \cdot \boldsymbol{e}_{\boldsymbol{y}}} d S
\end{aligned}
$$

where $\sigma_{p u}$ is the plastic threshold stress and is constant. $\boldsymbol{n}(\boldsymbol{X})$ and $\boldsymbol{t}(\boldsymbol{X})$ are the direction vectors for normal and frictional forces respectively.
The surfaces $A_{y}^{n}$ and $A_{y}^{t}$ are the projected contact areas along $\boldsymbol{e}_{y}$ direction, following the convention of Walraven, now extended for 3D.

Comparatively to the projected Eq. (9) in 2D, we now have to determine the contact patch $\mathscr{A}$ before calculating the integral in Eq. (15). Such a contact patch can be identified geometrically, as Walraven does in the 2D case. To this end, let us consider a rigid sphere of diameter $D$, as seen in Fig. 9a. The crack opening displacement is applied to a second spherical surface of same diameter, representing the opposite mortar/concrete face (represented in red on Fig. 9b). The region of overlapping/interlock between these two spheres is therefore remaining gray and measures $\mathscr{A}$.

The integration over the contact patch $\mathscr{A}$ is computed by discretizing the surface into $N^{e l}$ finite elements, each with an area $a_{e}$, a normal $\boldsymbol{n}_{e}$ and a tangent vector $\boldsymbol{t}_{e}$ as shown in Fig. 10. The tangent vector $t_{e}$ is defined as the unit vector mutually orthogonal to the normal $\boldsymbol{n}_{e}$ and a vector of the tangent plane normal to $\boldsymbol{e}_{y}$, i.e. $\boldsymbol{t}_{e} \propto\left(\boldsymbol{e}_{y} \wedge \boldsymbol{n}_{e}\right) \wedge \boldsymbol{n}_{e}$. Thus, the expression of the projected contact areas along $\boldsymbol{e}_{y}$ becomes:

$$
A_{y}^{n}=\sum_{e=1}^{N^{e l}} a_{e} \boldsymbol{n}_{e} \cdot \boldsymbol{e}_{\boldsymbol{y}} \quad \text { and } \quad A_{y}^{t}=\sum_{e=1}^{N^{e l}} a_{e} \boldsymbol{t}_{e} \cdot \boldsymbol{e}_{\boldsymbol{y}}
$$

Fig. 11 shows the evolution of the projected contact areas $\left(A_{y}^{n}\right.$ and $A_{y}^{t}$ ), normalised by their respective $A_{s p}$ as a function of normalised shear displacement for various displacement angles and sphere diameters. The material parameters $\sigma_{p u}$ and $\mu$ remain free parameters and are obtained by fitting the experimental results described in Section 3. The numerical model is thus fitted to each experimental result separately, using the method of least squares. Each fitting yields values of $\sigma_{p u}$ and $\mu$ which are recorded and later cross compared (refer to Appendix B). This procedure is used for fitting the concrete specimens as reported in this section and will also be used for the mortar specimens.

Fitting $\left(\sigma_{p u}, \mu\right)$ from Eq. (15) onto the displacement path, i.e. $\delta \in$ $[0 \mathrm{~mm}, 1.0 \mathrm{~mm}]$, yields regression curves revealing that this model

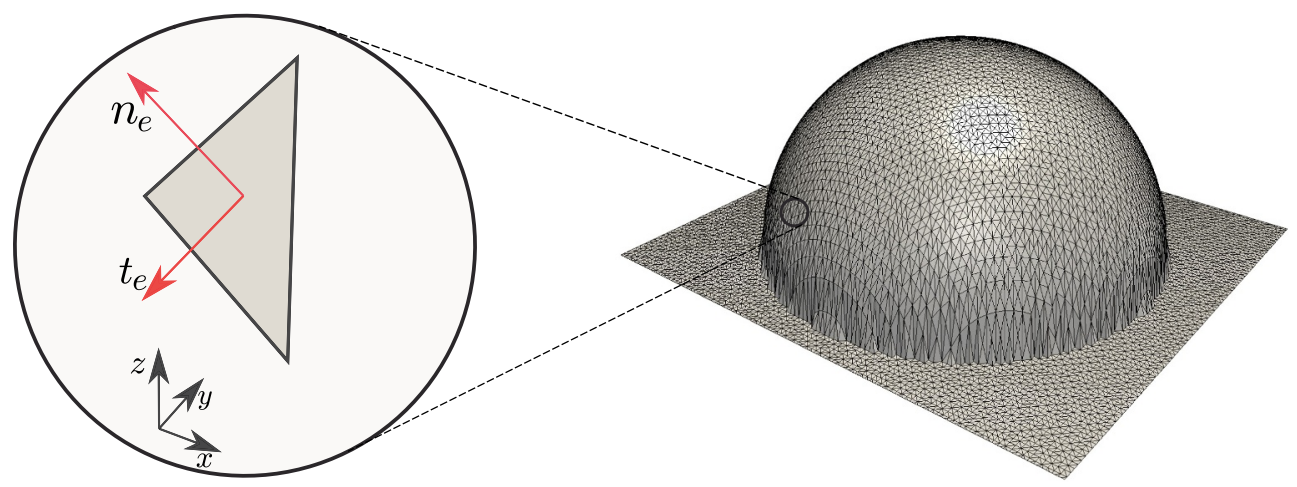

Fig. 10. A spherical aggregate discretize into finite elements. The zoom view shows the normal and tangential vector acting on a finite element. 

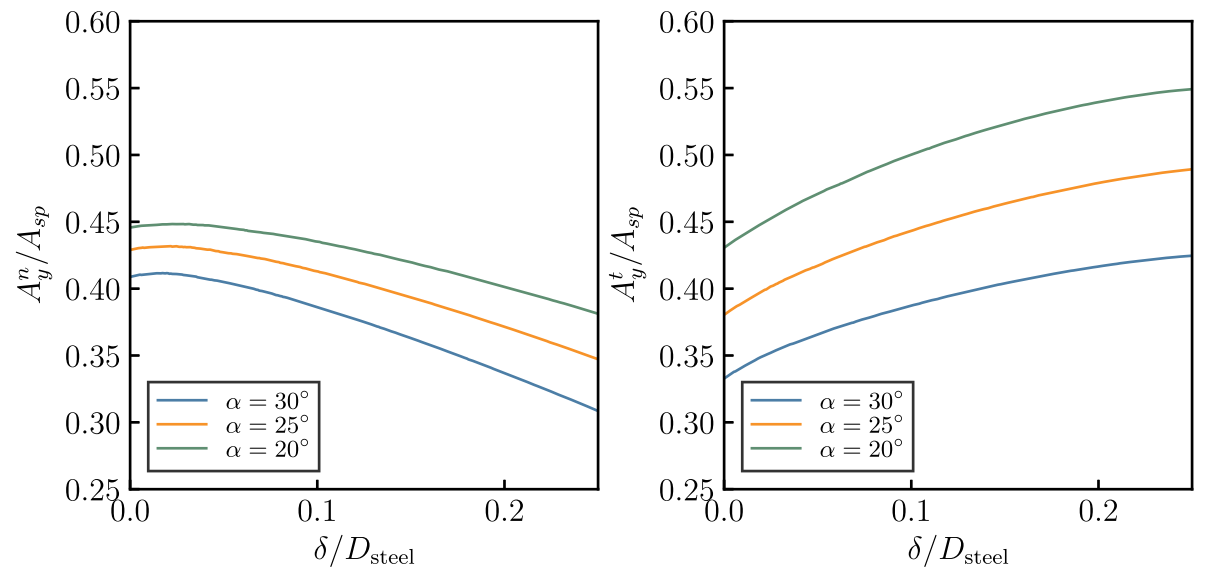

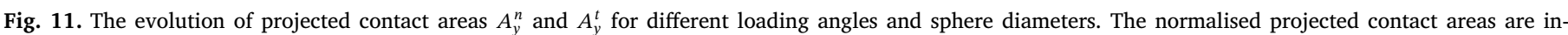
dependent of $D_{\text {steel. }}$.

cannot predict the entire shear resistance curve. This was expected because of the rigid perfectly-plastic assumption, whereas the actual onset of the experiment must be elasto-plastic. As a consequence, quite disparate values of both $\sigma_{p u}$ and $\mu$ are obtained by regression and even physically impossible negative values can be observed for $\mu$ (refer Table B.6). Thus, for analysis of the results, the value of the friction coefficient is set to a physically-consistent value according to Walraven ( $\mu=0.4$, [6]). Under this constraint, fitting only $\sigma_{p u}$ yields values that are acceptable both for concrete and mortar. However, Fig. 12 shows that the global behaviour cannot be captured. As a matter of facts, only an average behaviour roughly following the experimental curves can be obtained. Similar conclusions can be observed from fitting against the mortar specimens for the displacement path $(\delta \in[0 \mathrm{~mm}, 2.0 \mathrm{~mm}])$, as seen in Fig. 13. It is natural to charge this drawback onto the perfectlyplastic nature of the model, and to use it only for the softening parts of experimental results (the post-peak stress regions). Obtained regressions show a much better fit with experimental results. However, in all cases, the best fitted friction coefficients are negative and physically

(b) Free parameters: $\sigma_{p u}$

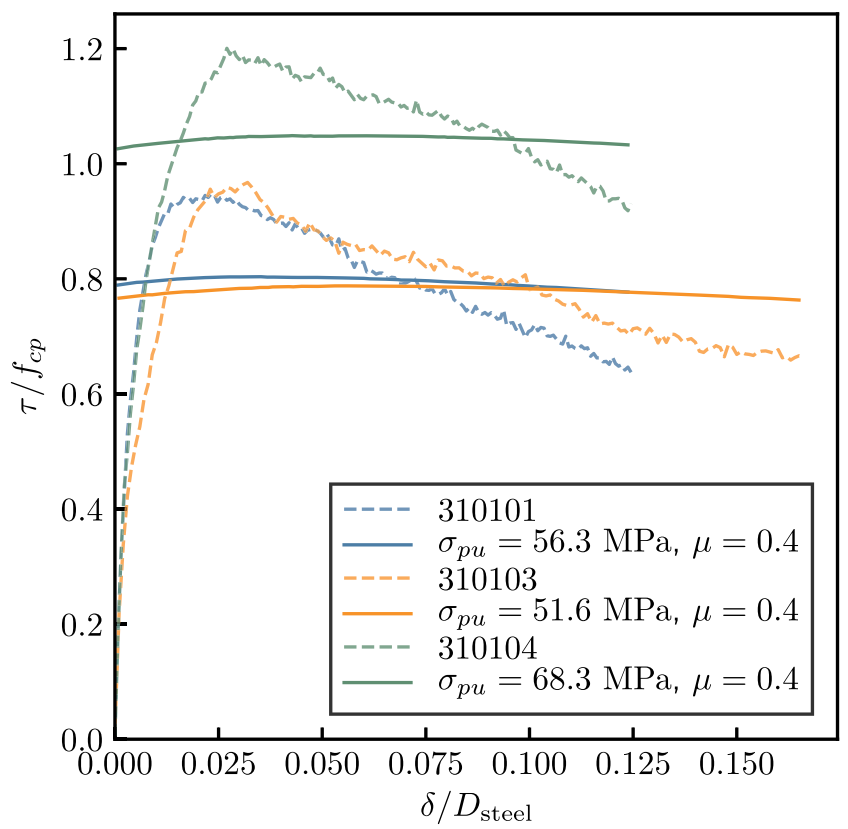

Fig. 12. Concrete specimens: Regression of Eq. (15) to experimental results for the entire shear displacement range $(\delta \in[0 \mathrm{~mm}, 1.0 \mathrm{~mm}])$ where only $\sigma_{p u}$ was fitted with a prescribed $\mu=0.4$. impossible (see Tables B.8, B.9). If $\mu$ is constrained to a fixed value, again the average behaviour may be captured leading to acceptable values for $\sigma_{p u}$, but the overall stress-resistance prediction curve remains inaccurate (see Figs. 14 and 15). This works very well for concrete samples, revealing that the presence of aggregates triggers an early onset of plastic flow in the matrix. With mortar samples, on the other end, the absence of confinement favors elasticity (or a delay in onset of plastic flow), which might explain the mismatch with the Two-Phase model.

The considerations concerning the obtained values of $\sigma_{p u}$ and $\mu$ demonstrate that the geometric model as described by Walraven is incomplete and this for two reasons. First, the hypothesis that the matrix's plastic behaviour dominates and the stress-strain relation of the matrix is rigid-perfectly plastic is not applicable for the entire load path: the initial loading phase (before the peak-stress) must be elasto-plastic. Secondly, one has to realize that if the matrix behaves as a perfectly plastic bulk during softening, the contact between matrix and aggregate cannot be deduced from a simple geometric intersection of the pristine

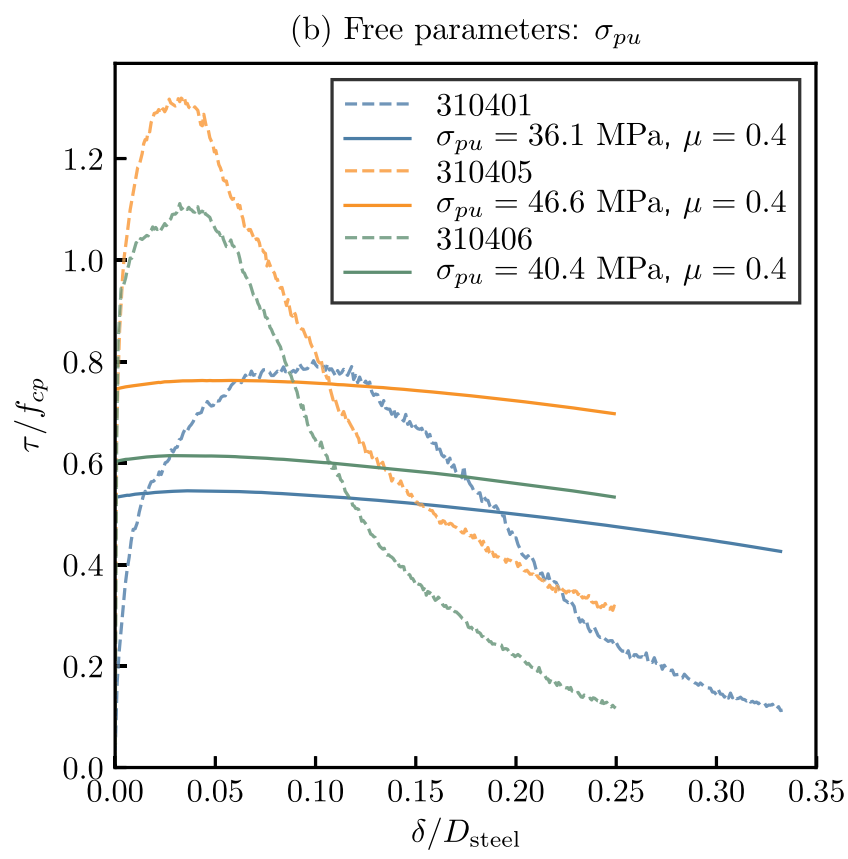

Fig. 13. Mortar specimens: Regression of Eq. (15) to experimental results for the entire shear displacement range $(\delta \in[0 \mathrm{~mm}, 2.0 \mathrm{~mm}])$ where only $\sigma_{p u}$ was fitted with a prescribed $\mu=0.4$. 
(b) Free parameters: $\sigma_{p u}$

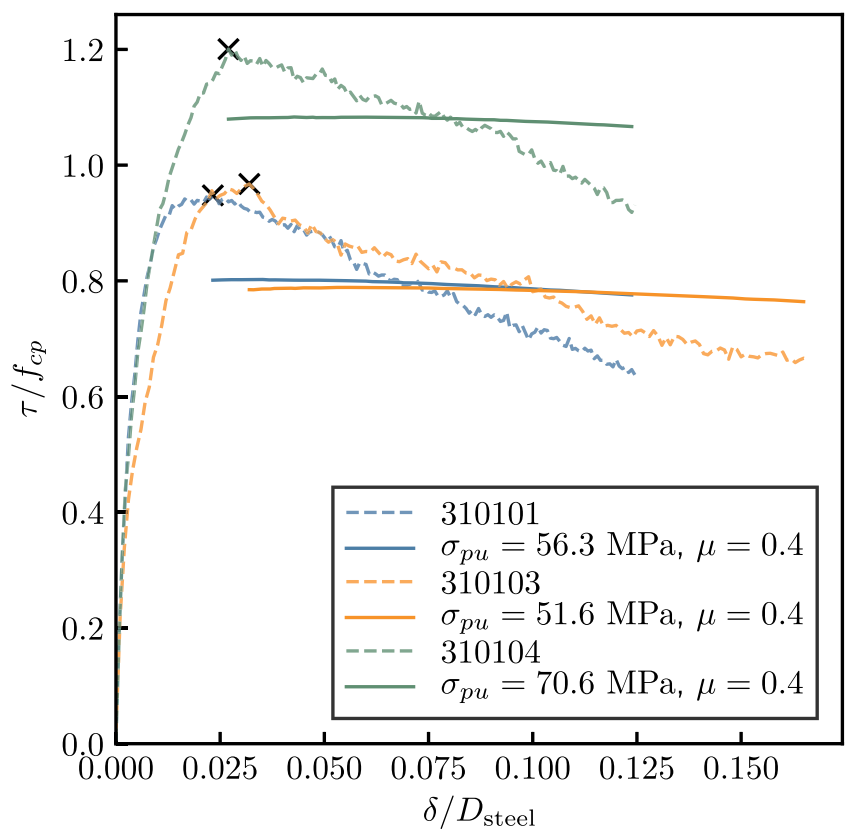

Fig. 14. Concrete specimens: Regression of Eq. (15) to experimental results for the post-peak shear displacement range $(\delta \in[0.2 \mathrm{~mm}, 1.0 \mathrm{~mm}])$ where only $\sigma_{p u}$ was fitted with a prescribed $\mu=0.4$.

(b) Free parameters: $\sigma_{p u}$

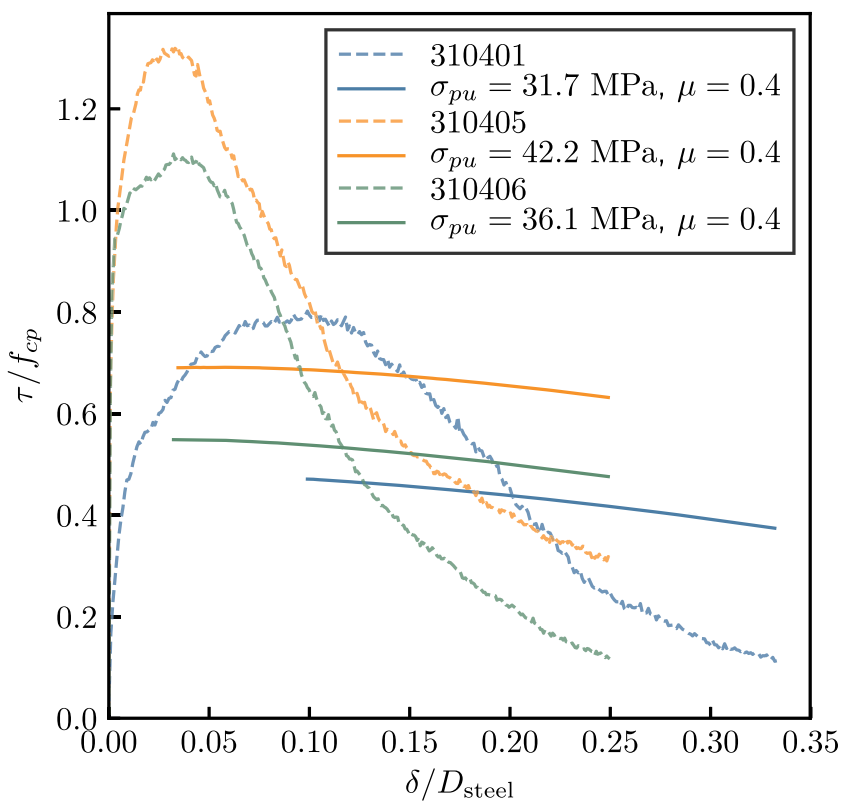

Fig. 15. Mortar specimens: Regression of Eq. (15) to experimental results for the post-peak shear displacement range where only $\sigma_{p u}$ was fitted with a prescribed $\mu=0.4$

geometries. This aspect has already been considered in some previous works $[13,14]$. In addition, the potential degradation of the matrix for very large displacements (as subjected to micro-crack development) is neglected in the softening phase. This is at the source of the incoherent predictions of the material parameter $\sigma_{p u}$. The ultimate goal of the present work is to introduce a variation of Walraven's method that addresses these issues, therefore allowing a description with physicallysound free parameters. These issues are addressed by $i$ ) accounting for the surface alterations after contact and ii) by introducing free parameters $\beta_{y}$ and $\beta_{z}$ to account for the elasto-plastic deformation and potential degradation of matrix during the initial loading phase and mimic its effect on perfectly-plastic regime.

\subsection{Two-Phase model enhancement: surface alterations}

A consistent modification of the Two-Phase model is proposed in this section to address the described inconsistencies. As previously stated, in such a regime, the mortar/concrete will deform substantially, so that the surface changes have to be taken into account to compute contact surfaces.

To account for this, a possible strategy considers that the deformation of contact area can again be approximated in a purely geometric sense. The idea is to remove any geometric interpenetration between mortar and aggregate bodies created by the imposed homogeneous displacement $(\delta, w)$. Such a geometric configuration is illustrated in Fig. 16a. Because mostly mortar will undergo a plastic flow, only its surface is modified by the vertical projection onto the aggregate surface. Such a projection is done at each incremental step. Fig. 16b shows the evolution of the contact area with and without considering such a plastic alteration of the surface. Remarkably, the corrected contact area now decreases as a function of $\delta$.

The contact patch $\mathscr{A}$ is deformed as soon as the shear displacement reaches the value corresponding to the peak stress. Fig. 17 shows the evolution of projected contact areas after taking deformation into consideration. With the corrected projected contact areas ( $A_{y}^{n}$ and $\left.A_{y}^{t}\right)$, the regression procedure presented in Section 4.1 can be employed again.

The regression is done by considering $\sigma_{p u}$ as free parameter with $\mu$ fixed to 0.4 (trying to fit both $\sigma_{p u}$ and $\mu$ leads potentially to un-physical values similar to the ones presented in the previous section). Figs. 18 and 19 shows the regression curves for both concrete and mortar specimens.

\subsection{Two-Phase model enhancement: elasto-plastic deformation of matrix}

Fitting Eq. (15) with corrected contact area still gives a mismatch with experimental stress-displacement curves. The origin of this discrepancy is the projection strategy employed, which only crudely account for plastic deformations due to contact forces, and therefore leads to inaccurate contact areas. During the pre-peak regime, where elastoplastic deformation occurs, only some portion of the contact surface will plastify to take the shape of the steel aggregate. To illustrate this point, we take an example of a steel aggregate indenting an elastoplastic material as shown in Fig. 20i. Allowing for interpenetration of bodies, the contact area $\mathscr{A}$ considered, based on our vertical projection strategy, is shown in Fig. 20i-b in red. However, due to elasto-plastic deformation the actual deformed profile will be different and the actual contact area $\mathscr{A}^{*}$ may be much smaller as shown in the Fig. 20i-c. Fig. 20ii shows the elasto-plastic deformation of matrix and the correction of the contact area for the geometry considered in our case.

Therefore, the actual contact area will be less than the contact area computed from our projection strategy. At the peak stress, when elastoplastic regime transitions to perfectly-plastic regime, the actual contact area $\mathscr{A}^{*}$ will need a correction factor.

$\mathscr{A}^{*}=\mathscr{A}-\mathscr{A}_{e p}$

where $\mathscr{A}$ is the contact area computed from vertical projection and $\mathscr{A}_{e p}$ is the correction contact area, to be computed at the transition to perfectly plastic regime i.e. at peak stress. Eq. (15) is then modified to account for the true contact area. 


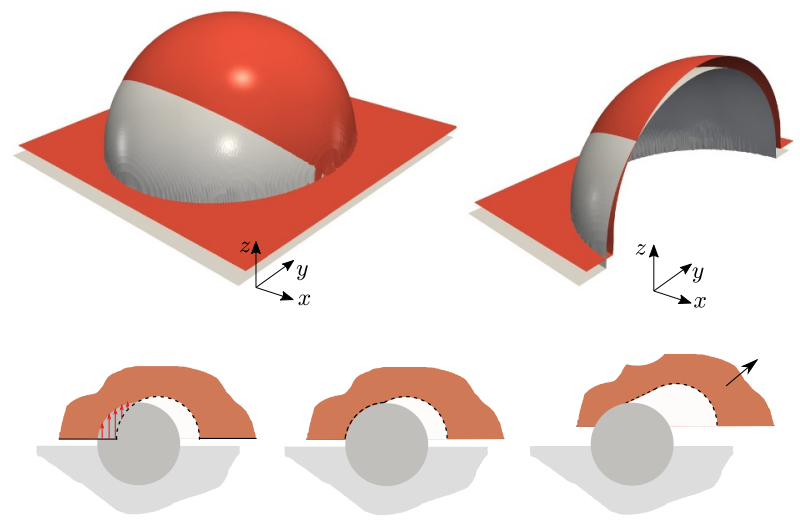

(a)

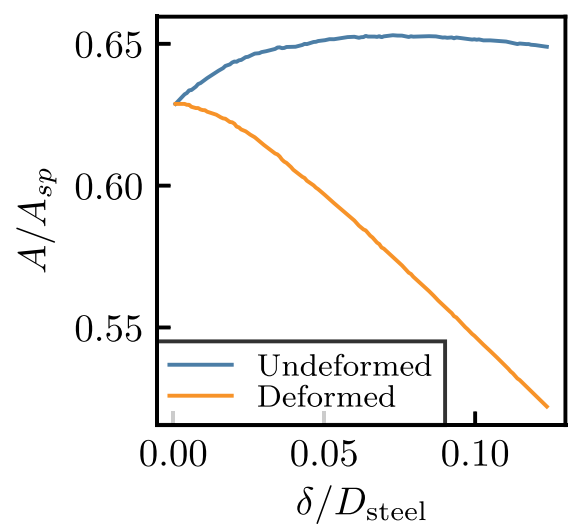

(b)

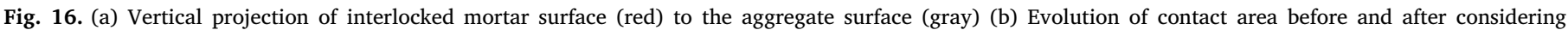

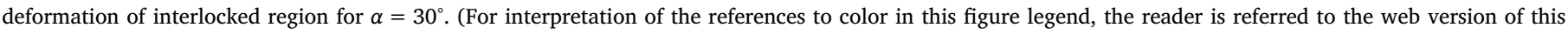
article.)

$$
\begin{aligned}
F\left(\mathscr{A}^{*}(w, \delta)\right) & =F\left(\mathscr{A}(w, \delta)-\mathscr{A}_{e p}\right) \\
& =\int_{\mathscr{A}} \tau^{\boldsymbol{P}}(\boldsymbol{X}) \cdot \boldsymbol{e}_{y} d S-\int_{\mathscr{d e p}} \tau^{\boldsymbol{P}}(\boldsymbol{X}) \cdot \boldsymbol{e}_{y} d S \\
& \left.=\sigma_{p u} \int_{\mathscr{A}} \underset{A_{y}^{n}}{\boldsymbol{n}(\boldsymbol{X}) \cdot \boldsymbol{e}_{y}} d S+\sigma_{p u} \mu \int_{\mathscr{A}} \boldsymbol{t} \underset{A_{y}^{t}}{(\boldsymbol{X})}\right) \cdot \boldsymbol{e}_{\boldsymbol{y}} d S-\sigma_{p u} \mathscr{A}_{e p, y}
\end{aligned}
$$

where $\mathscr{A}_{e p, y}$ is the projection of the correction contact area $\mathscr{A}_{e p}$ along $\boldsymbol{e}_{\boldsymbol{y}}$ direction. Eq. (15) can be modified to compute the normal forces by taking the projection along $\boldsymbol{e}_{z}$ direction:

$$
\begin{aligned}
\left\langle F_{N}\right\rangle & =\int_{\mathscr{A}} \tau^{\boldsymbol{P}}(\boldsymbol{X}) \cdot \boldsymbol{e}_{z} d S-\int_{\mathscr{A} e p} \tau^{\boldsymbol{P}}(\boldsymbol{X}) \cdot \boldsymbol{e}_{z} d S \\
& =\sigma_{p u} \int_{\mathscr{A}} \boldsymbol{n} \underset{A_{z}^{n}}{\boldsymbol{n}(\boldsymbol{X}) \cdot \boldsymbol{e}_{z}} d S+\sigma_{p u} \mu \underset{\mathscr{A}}{\int_{A_{z}^{t}} \boldsymbol{t}(\boldsymbol{X}) \cdot \boldsymbol{e}_{z}} d S-\sigma_{p u} \mathscr{A}_{e p, z}
\end{aligned}
$$

where $A_{z}^{n}, A_{z}^{t}$ and $\mathscr{A}_{e p, z}$ are areas projected along $\boldsymbol{e}_{z}$ direction. We assume that the corrected contact areas $\mathscr{A}_{e p, y}$ and $\mathscr{A}_{e p, z}$ are proportional to the $\mathscr{A}$ projected along $\boldsymbol{e}_{y}$ and $\boldsymbol{e}_{z}$ and can be correlated by introducing parameter $\beta_{y}$ and $\beta_{z}$ :

$$
\begin{aligned}
\mathscr{A}_{e p, y} & \propto \mathscr{A}(\boldsymbol{n}(\boldsymbol{X})+\mu \boldsymbol{t}(\boldsymbol{X})) \cdot \boldsymbol{e}_{y} \\
& =\beta_{y} \mathscr{A}(\boldsymbol{n}(\boldsymbol{X})+\mu \boldsymbol{t}(\boldsymbol{X})) \cdot \boldsymbol{e}_{y} \\
& =\beta_{y}\left(A_{y}^{n}+\mu A_{y}^{t}\right)
\end{aligned}
$$

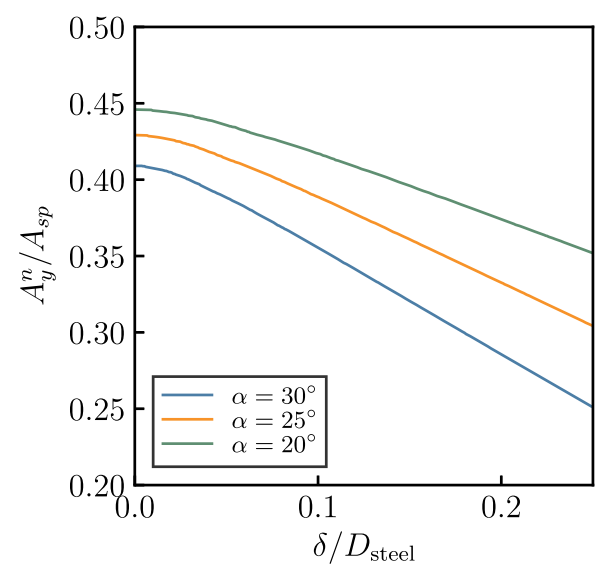

$$
\begin{aligned}
\mathscr{A}_{e p, z} & \propto \mathscr{A}(\boldsymbol{n}(\boldsymbol{X})+\mu \boldsymbol{t}(\boldsymbol{X})) \cdot \boldsymbol{e}_{z} \\
& =\beta_{z} \mathscr{A}(\boldsymbol{n}(\boldsymbol{X})+\mu \boldsymbol{t}(\boldsymbol{X})) \cdot \boldsymbol{e}_{z} \\
& =\beta_{z}\left(A_{z}^{n}+\mu A_{z}^{t}\right)
\end{aligned}
$$

where $A_{y}^{n}, A_{y}^{t}$ and $A_{z}^{n}, A_{z}^{t}$ are calculated at $\left\{\delta_{y}=\arg \max \tau(\delta)\right\}$ and at $\left\{\delta_{z}=\arg \max \sigma(\delta)\right\}$ respectively. As can be observed from the above equations $\mathscr{A}_{e p, y}$ and $\mathscr{A}_{e p, z}$ depend on the loading angle $\alpha$, the diameter of the sphere $D_{\text {steel }}$ and the parameters $\beta_{y}, \beta_{z}$.

This extended numerical model is again fitted to each experimental result separately using the method of least squares. We fit Eq. (18) to the experimental tangential forces to obtain regression values of $\sigma_{p u}$ and $\beta_{y}$. The obtained value of $\sigma_{p u}$ is then used for fitting Eq. (19) to the experimental normal forces with $\beta_{z}$ as the only free parameter. Fig. 21 shows the regression curves for a fixed value of $\mu=0.4$ and $\sigma_{p u}, \beta_{y}$ and $\beta_{z}$ as free parameters for concrete specimens.As can be seen, the postpeak behaviour is captured accurately for concrete specimens. The values computed for $\sigma_{p u}$ of samples 310102 and 310201 (see Table 4) are considered as outliers. As discussed in Section 3.2, for test 310102 the shear force starts increasing in the softening phase and for test 310201 , a large plateau is reached (behaviour attributed to the sphere coming in contact with a large aggregate). The other values obtained for $\sigma_{p u} / f_{c p}$ are, for the majority of the cases, in the range of values 3 to 4 . Also, the values of $\beta_{y}$ and $\beta_{z}$ obtained from two different fittings are consistent for a given geometry and loading angle, typically between 0.5 and 0.8 . A detailed discussion is presented in next section about the values obtained for $\sigma_{p u}, \beta_{y}$ and $\beta_{z}$. Concerning the mortar specimens, the

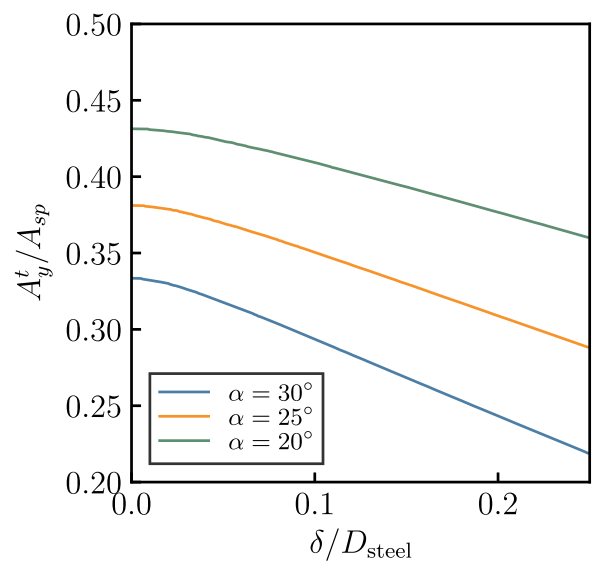

Fig. 17. The evolution of projected contact areas $A_{y}^{n}$ and $A_{y}^{t}$ after considering deformation of interlocked region for different loading angles and sphere diameters. 
(b) Free parameters: $\sigma_{p u}$

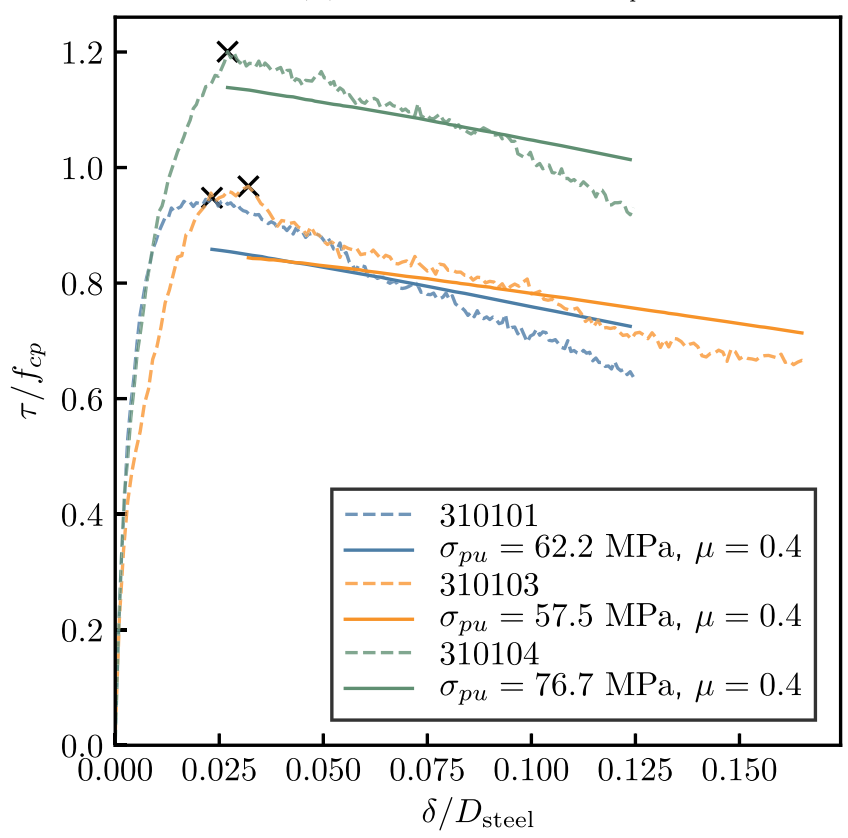

Fig. 18. Concrete specimens: Regression of Eq. (15) to experimental results for the post-peak shear displacement range considering deformation of contact area where only $\sigma_{p u}$ was fitted with a prescribed $\mu=0.4$.

(b) Free parameters: $\sigma_{p u}$

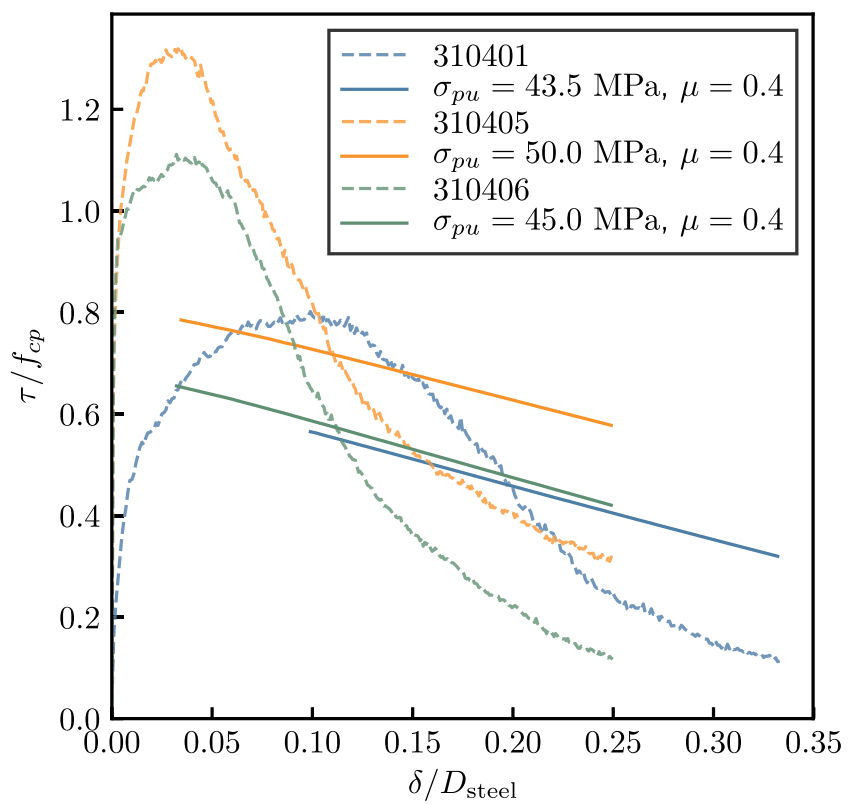

Fig. 19. Mortar specimens: Regression of Eq. (15) to experimental results for the post-peak shear displacement range considering deformation of contact area where only $\sigma_{p u}$ was fitted with a prescribed $\mu=0.4$.

assumption that the perfectly-plastic regime starts right after the peak stress does not seem to hold (see Fig. 22) and two post-peak regimes seem to develop. Fitting Eq. (18) just after the peak stress yields too large values of $\sigma_{p u}$. This discrepancy can be explained by the fact that the mortar has a less plastic response than concrete (the plastification allowing to measure the effective $\sigma_{p u}$ is only reached at a late stage, $\approx$ $1.0 \mathrm{~mm}$ ). This seems consistent with the experimental results when the fitting starts after $1.0 \mathrm{~mm}$ of displacement: the values of $\sigma_{p u}$ are thus similar to what was obtained for concrete specimens, which were made of mortar with a similar compressive strength (but with larger scatter of the results). It is also interesting to note that the values of $\beta_{y}$ and $\beta_{z}$ are consistent with each other in all cases.

\section{Discussion}

The values of $\sigma_{p u}$ predicted by the previously presented approach yield valuable information allowing to interpret its nature in Two-Phase models. As presented in Tables 4 and $5, \sigma_{p u} / f_{c p}$ is in the same order of magnitude as the ratio observed in various studies. For instance, the analytical works [26] and [27] find $\sigma_{p u}=3 f_{c p}$ in the rigid-plastic regime during the indentation of an elastic-plastic half-space by a spherical rigid body. Also, for concrete it is observed that a highly confined matrix should produce a higher effective strength, leading to much larger values of $\sigma_{p u} / f_{c p}[28,29]$. Afterall, the proportionality factor between $\sigma_{p u}$ and $f_{c p}$ depends on the heterogeneity of the bulk and on the shape of the contacting surfaces.

Even if $\sigma_{p u}$ is taken equal to $f_{c p}$, Two-Phase models may provide reasonable estimates of the peak tangential resistance in real cases with rough cracked surfaces. This paradox can be explained with the introduction of the statistics, which may compensate for the missing proportionality factor. Let us recall the central equation of Two-Phase models, as written by Walraven:

$\left\langle F_{R}\right\rangle=\iint \Psi(D, h) F(D, h) d D d h$

where $\Psi(D, h)$ is the probability density of circular aggregates of diameter $D$ being intersected at elevation $h$, and $F(D, h)$ is the force contribution of this particular geometrical situation. The strategy of Walraven was to write $\Psi(D, h)=\lambda(D) \rho(D, h)$ as a product between the probability density $\lambda(D)$ of having an aggregate of diameter $D$ and the probability density $\rho(D, h)$ of cutting such an aggregate at elevation $h$. A first source of inaccuracy comes from the $2 \mathrm{D}$ projection the force $F(D, h)$, assuming that every slice of matter is not exchanging forces with its the surrounding. Secondly, Walraven assumed that $\rho(D, h)=2 / D$ which seems to be an adhoc function decaying smoothly with the asperity radius. Both these points may lead to a hidden modification of the proportionality factor $\sigma_{p u} / f_{c p}$, explaining how the peak shear resistance could be captured with a wrong $\sigma_{p u}$ value.

Nevertheless, it was demonstrated that obtaining the correct behaviour along the entire loading path needs an accurate prediction of the contact area. Without a full resolution of the mechanical problem, an area correction had to be introduced, $\mathscr{A}_{e p}$, which represents the error in our geometrically-based contact area prediction. This obviously neglected the elasto-plastic onset deformation of the matrix. This error will depend upon $f_{c p}$ and on the arrangement of aggregates around the steel spheres. Indeed, aggregates close to the interlock contact may create obstacles which would trigger early plastic flows in the matrix, therefore leading to small values of the correction area $\mathscr{A}_{e p}$.

All the raised points call for computations resolving explicitly the plastic deformation occurring in the bulk. Only by using large scale finite elements, with fracture, plasticity and contact algorithms, will it be possible to obtain the accurate evolution of contact area and forces, and therefore would enhance Two-Phase models predictive capacity. Some more fundamental modelling difficulties are still to be accounted for statistical Two-Phase models to describe aggregate interlocking in cracked concrete:

- Aggregates are usually not spherical [30].

- Failures in structures may be triggered by the limited tensile capacity of the matrix, which will therefore develop cracks and thus present a reduced strength [2].

- The range of aggregate sizes to be considered as part of the matrix (and not as aggregate) is not precisely defined.

- Cracks are not straight planes, but have an undulated shape which additionally influences the overall roughness [3]. 
(i)
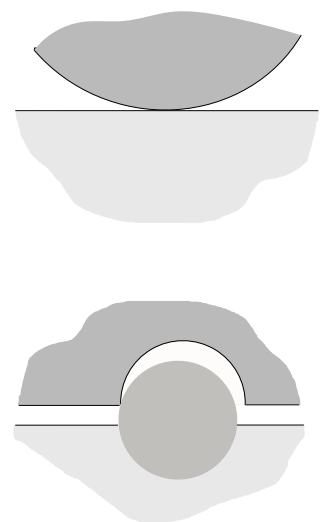

(a)
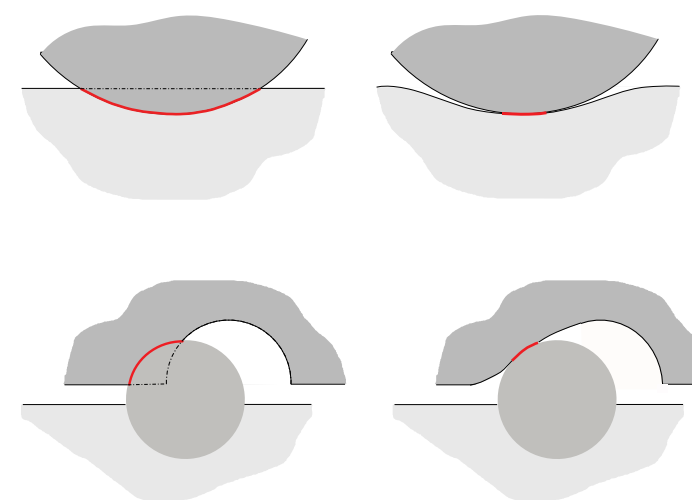

(b)

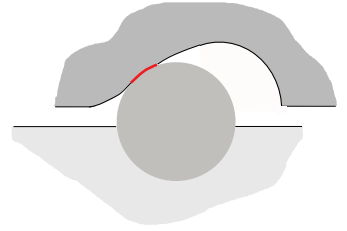

(c)

Fig. 20. (a) Indentation of an elasto-plastic material by steel aggregate (b) Contact area ( $A$ ) (in red) computed from vertical projection strategy (c) Actual contact area $\mathscr{A}^{*}$ (in red) due to elasto-plastic deformation of the material. (For interpretation of the references to color in this figure legend, the reader is referred to the web version of this article.)

\section{Conclusions}

Two-Phase models can predict the shear resistance due to asperity interlock. They encompass a statistical treatment, with a probability density function of interlock situations, and geometrically computed forces as key ingredients. In the past, such theories considered 2D approximations. A 3D extension to the force evaluation has been developed to predict the shear resistance measured during several experiments of well-defined interlock configurations, where statistics can be omitted: concrete and mortar samples have been loaded against three steel spheres in order to stay close to the assumptions of Two-Phase models, i.e. flat crack plane and spherical aggregates. The main conclusions of this investigation are listed below:

1. For all the presented experiments, both concrete and mortar, the maximum interlock normal/shear stress (i.e. the normal/shear strengths) normalised by the equivalent plastic resistance $\left(f_{c p}\right)$ are shown to decrease with increasing loading angle $(\alpha)$. However, the load-displacement curves differ strongly. Concrete exhibits a larger scatter, and a generally smaller yield strength. This larger scatter can be explained by the presence of large aggregates within the vicinity of interlocking regions.

2. Walraven's hypothesis of perfectly-plastic deformation of the matrix
Table 4

Concrete specimens: Obtained values of $\sigma_{p u}, \beta_{y}$ and $\beta_{z}$ considering contact patch deformation during elasto-plastic regime.

\begin{tabular}{llllllll}
\hline \multicolumn{7}{l}{ Fitted: Concrete specimens } \\
\hline$\#$ & $\alpha\left[^{\circ}\right]$ & $D_{\text {steel }}[\mathrm{mm}]$ & $\sigma_{p u}[\mathrm{MPa}]$ & $\sigma_{p u} / f_{c p}[-]$ & $\mu[-]$ & $\beta_{y}[-]$ & $\beta_{z}[-]$ \\
\hline 310101 & 30 & 8 & 140.91 & 3.64 & 0.4 & 0.52 & 0.64 \\
310102 & 25 & 6 & 205.29 & 5.30 & 0.4 & 0.73 & 0.55 \\
310103 & 25 & 6 & 121.43 & 3.14 & 0.4 & 0.49 & 0.56 \\
310104 & 25 & 8 & 157.62 & 4.07 & 0.4 & 0.49 & 0.57 \\
310201 & 25 & 6 & 469.05 & 12.30 & 0.4 & 0.85 & 0.86 \\
310202 & 20 & 6 & 104.66 & 2.74 & 0.4 & -0.39 & 2.59 \\
310302 & 30 & 8 & 158.31 & 3.22 & 0.4 & 0.62 & 0.67 \\
310304 & 25 & 6 & 186.95 & 3.80 & 0.4 & 0.29 & 0.23 \\
\hline
\end{tabular}

is not applicable during the initial loading stage for both concrete and mortar. This is justified by the fact that the onset of deformation is necessarily elasto-plastic. A clear plastic behaviour is only completely developed for relatively large penetrations of the aggregates in the matrix (in any case after the shear stress peak).

3. It is demonstrated that strictly adopting Walraven's modelling assumptions leads to un-physical values of the plastic threshold stress $\left(\sigma_{p u}\right)$ and the friction coefficient $(\mu)$. This is obtained by numerical

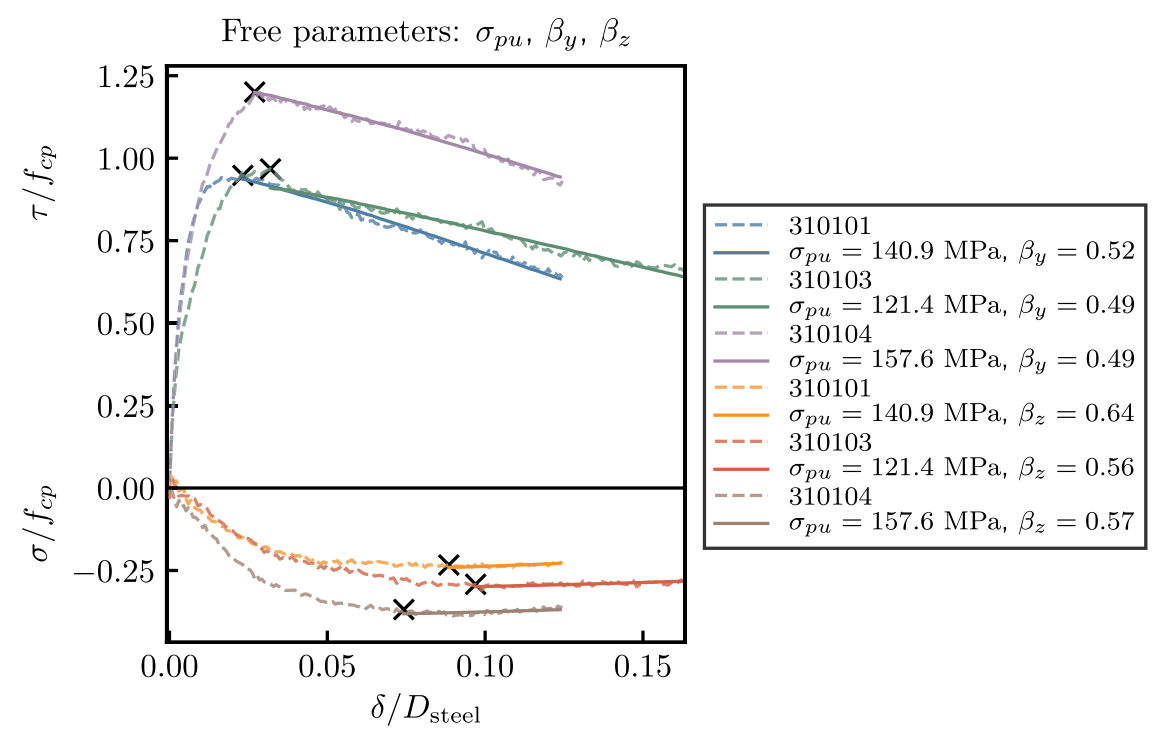

Fig. 21. Concrete specimens: Regression of Eqs. (18) and (19) to experimental results considering contact patch deformation during elasto-plastic regimes. 


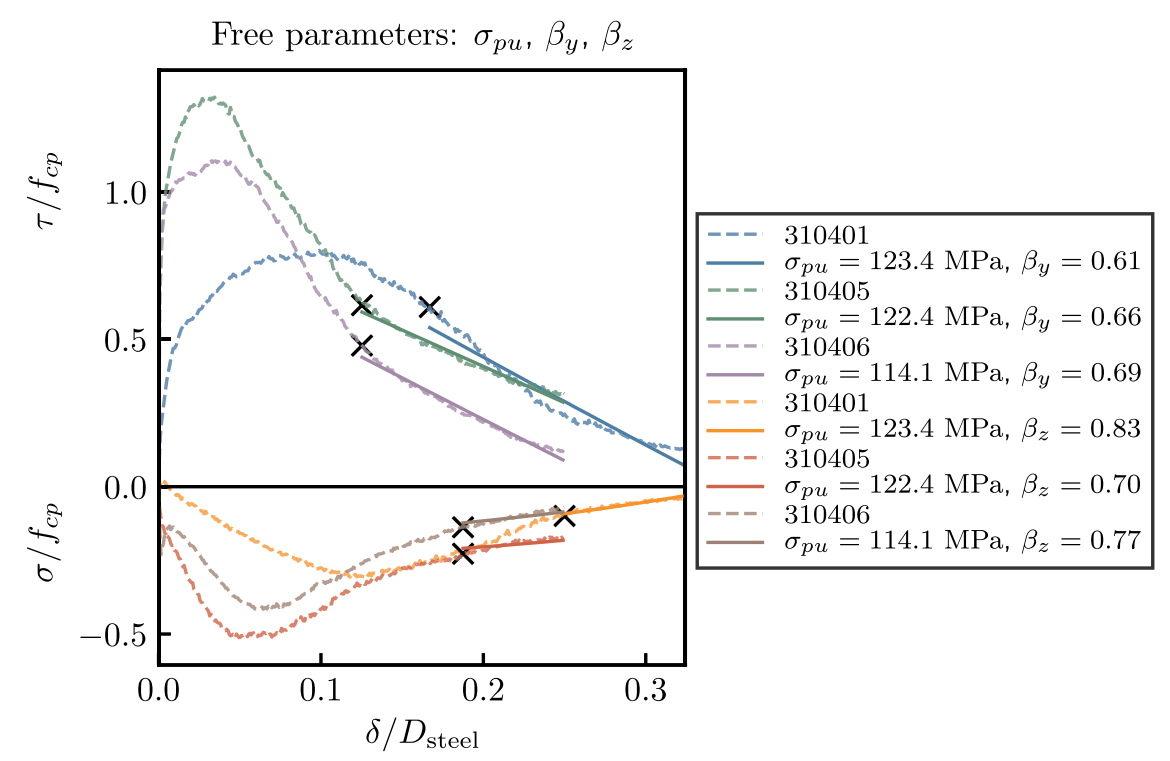

Fig. 22. Mortar specimens: Regression of Eqs. (18) and (19) to experimental results considering contact patch deformation during elasto-plastic regimes.

Table 5

Mortar specimens: Obtained values of $\sigma_{p u}, \beta_{y}$ and $\beta_{z}$ considering contact patch deformation during elasto-plastic regime.

\begin{tabular}{llllllll}
\hline \multicolumn{7}{l}{ Fitted: Mortar specimens } \\
\hline$\#$ & $\alpha\left[^{\circ}\right]$ & $D_{\text {steel }}[\mathrm{mm}]$ & $\sigma_{p u}[\mathrm{MPa}]$ & $\sigma_{p u} / f_{c p}[-]$ & $\mu[-]$ & $\beta_{y}[-]$ & $\beta_{z}[-]$ \\
\hline 310401 & 30 & 6 & 123.41 & 3.40 & 0.4 & 0.61 & 0.83 \\
310402 & 20 & 6 & 328.01 & 9.03 & 0.4 & 0.77 & 0.74 \\
310404 & 20 & 8 & 188.80 & 5.20 & 0.4 & 0.56 & 0.49 \\
310405 & 25 & 8 & 122.41 & 3.37 & 0.4 & 0.66 & 0.70 \\
310504 & 15 & 8 & 328.76 & 7.80 & 0.4 & 0.57 & 0.32 \\
310505 & 20 & 8 & 171.44 & 4.07 & 0.4 & 0.43 & 0.24 \\
310506 & 30 & 8 & 130.12 & 3.09 & 0.4 & 0.79 & 0.79 \\
310406 & 30 & 8 & 114.11 & 3.14 & 0.4 & 0.69 & 0.77 \\
\hline
\end{tabular}

regression of the Two-Phase model onto the experimental results, which yields negative values of the friction coefficient. Upon constraining $\mu=0.4$ (as performed by Walraven) and applying the regression only for the post-peak regime, physically-consistent values of $\sigma_{p u}$ are obtained. This yields however to a significant mismatch with the stress-displacement experimental curves.

4. The novel approach proposed in this paper, allows to obtain a matching post-peak response (dominated by the plastic behaviour of the material), by refining the contact areas employed by the model. First, the contacting area should account for the residual changes in the matrix surface due to plastic deformation during the loading path. This effect globally reduces the contact area for an increasing displacement (which was not accounted in the original Two-Phase model). Secondly, the elastic-plastic deformation of the matrix should be accounted for in order to further improve the measure of the contact area. A scalar correction factor $\left(\mathscr{A}_{e p}\right.$ ) has been introduced, yielding to very good agreements with all the experimental results.

It was also shown that $\mathscr{A}_{e p}$ is proportional to the geometrically evaluated contact area, with $\beta$ a proportionality factor, ranging from 0.5 up to 0.8 .

5. Contrary to common usage of Walraven's theory, assuming $\sigma_{p u}=f_{c p}$, it was shown that the proportionality factor between $\sigma_{p u} / f_{c p}$ is not constant. In order to predict the entire stress-strain curve (and not only the peak value of the shear resistance), the proportionality factor must be known. This remains difficult as it requires an accurate prediction of the interlocking contact areas, only accessible with the complete resolution of the mechanical problem (for instance with large scale finite elements techniques, involving fracture, plasticity and contact algorithms).

\section{Acknowledgments}

This work has been funded by the Swiss National Science Foundation, research grant 200021_169649. The authors are very appreciative of the support received.

\section{Appendix A. Hurst exponent for Walraven's surface}

A numerical concrete sample is generated for a given packing density $\left(p_{k}\right)$ and an aggregate range ( $\left.\left[D_{\min }, D_{\max }\right]\right)$ using placing algorithm proposed in Ref. [31]. The aggregates were assumed to be spherical in shape and the fuller distribution curve was used for distribution of aggregates. The numerically fabricated concrete sample is cut at an arbitrarily plane according to the geometrical hypothesis of Two-Phase model to generate an idealized crack plane Fig. A.23a. Fig. A.23b shows the Power Spectral Density [32, 33] and corresponding Hurst exponent for such a surface. However, it should be noted that Walraven chose $D_{\min }=0.25 \mathrm{~mm}$, while it was set to $2 \mathrm{~mm}$ for this study. Similarly, the packing density $p_{k}$ used in Walraven's original model is around $70 \%$ while it is $60 \%$ for this study. 


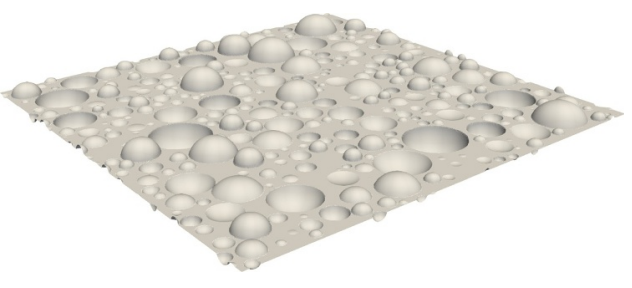

(a)

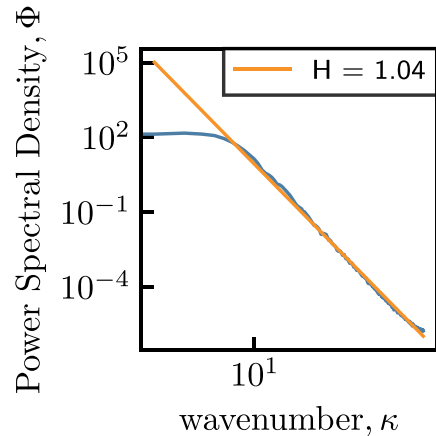

(b)

Fig. A.23. (a) Idealized crack surface for $60 \%$ packing density and diameters in range [14 mm, 2 mm]. (b) Hurst exponent $H$ for surface in (a).

\section{Appendix B. Detailed results}

Tables B.6- B.11 present the values of $\sigma_{p u}$ and $\mu$ obtained for the various concrete and mortar specimens. Not all tests have been considered, as 310303 and 310305 where subjected to special kinematics and tests 310204, 310301 and 310302 had no clearly identified softening phase. Similarly, the mortar specimens 310403, 310502 and 310503 have been omitted due to their anomalous behaviour during after the peak.

\section{B.1. Review of original formulation according to Walraven}

Table B.6

Concrete specimens: Obtained values of $\sigma_{p u}$ and $\mu$ for concrete specimens when fitted over the shear displacement range $(\delta \in[0 \mathrm{~mm}, 1.0 \mathrm{~mm}])$.

\begin{tabular}{|c|c|c|c|c|c|c|c|c|}
\hline \multirow[b]{2}{*}{ \# } & \multirow[b]{2}{*}{$\alpha\left[^{\circ}\right]$} & \multirow[b]{2}{*}{$D_{\text {steel }}[\mathrm{mm}]$} & \multicolumn{3}{|c|}{ Fitted: $\sigma_{p w} \mu$} & \multicolumn{3}{|l|}{ Fitted: $\sigma_{p u}$} \\
\hline & & & $\sigma_{p u}[\mathrm{MPa}]$ & $\sigma_{p u} / f_{c p}[-]$ & $\mu[-]$ & $\sigma_{p u}[\mathrm{MPa}]$ & $\sigma_{p u} / f_{c p}[-]$ & $\mu[-]$ \\
\hline 310101 & 30 & 8 & 124.55 & 3.22 & -0.4 & 56.35 & 1.46 & 0.4 \\
\hline 310102 & 25 & 6 & -68.41 & -1.77 & -2.0 & 52.30 & 1.35 & 0.4 \\
\hline 310103 & 25 & 6 & 96.37 & 2.49 & -0.2 & 51.56 & 1.33 & 0.4 \\
\hline 310104 & 25 & 8 & 51.73 & 1.34 & 0.8 & 68.32 & 1.76 & 0.4 \\
\hline 310201 & 25 & 6 & -114.42 & -3.00 & -1.5 & 49.45 & 1.30 & 0.4 \\
\hline 310202 & 20 & 6 & -88.62 & -2.32 & -2.7 & 122.53 & 3.21 & 0.4 \\
\hline 310302 & 30 & 8 & 263.43 & 5.35 & -0.8 & 52.55 & 1.07 & 0.4 \\
\hline 310304 & 25 & 6 & 93.19 & 1.89 & 0.7 & 112.40 & 2.28 & 0.4 \\
\hline
\end{tabular}

Table B.7

Mortar specimens: Obtained values of $\sigma_{p u}$ and $\mu$ when fitted over the shear displacement range $(\delta \in[0 \mathrm{~mm}, 2.0 \mathrm{~mm}])$.

\begin{tabular}{|c|c|c|c|c|c|c|c|c|}
\hline \multirow[b]{2}{*}{$\#$} & \multirow[b]{2}{*}{$\alpha\left[^{\circ}\right]$} & \multirow[b]{2}{*}{$D_{\text {steel }}[\mathrm{mm}]$} & \multicolumn{3}{|c|}{ Fitted: $\sigma_{p u}, \mu$} & \multicolumn{3}{|l|}{ Fitted: $\sigma_{p u}$} \\
\hline & & & $\sigma_{p u}[\mathrm{MPa}]$ & $\sigma_{p u} / f_{c p}[-]$ & $\mu[-]$ & $\sigma_{p u}[\mathrm{MPa}]$ & $\sigma_{p u} / f_{c p}[-]$ & $\mu[-]$ \\
\hline 310401 & 30 & 6 & 119.83 & 3.30 & -0.5 & 36.11 & 0.99 & 0.4 \\
\hline 310402 & 20 & 6 & 282.54 & 7.77 & -0.4 & 86.37 & 2.38 & 0.4 \\
\hline 310404 & 20 & 8 & 176.16 & 4.85 & -0.3 & 74.53 & 2.05 & 0.4 \\
\hline 310405 & 25 & 8 & 257.54 & 7.09 & -0.7 & 46.63 & 1.28 & 0.4 \\
\hline 310505 & 20 & 8 & 221.93 & 5.26 & -0.3 & 99.03 & 2.35 & 0.4 \\
\hline 310506 & 30 & 8 & 281.88 & 6.68 & -0.7 & 48.86 & 1.16 & 0.4 \\
\hline 310406 & 30 & 8 & 246.59 & 6.79 & -0.7 & 40.45 & 1.11 & 0.4 \\
\hline
\end{tabular}

Table B.8

Concrete specimens: Obtained values of $\sigma_{p u}$ and $\mu$ for concrete specimens when fitted over the post-peak shear displacement range.

\begin{tabular}{|c|c|c|c|c|c|c|c|c|}
\hline \multirow[b]{2}{*}{$\#$} & \multirow[b]{2}{*}{$\alpha\left[^{\circ}\right]$} & \multirow[b]{2}{*}{$D_{\text {steel }}[\mathrm{mm}]$} & \multicolumn{3}{|c|}{ Fitted: $\sigma_{p u} \mu$} & \multicolumn{3}{|l|}{ Fitted: $\sigma_{p u}$} \\
\hline & & & $\sigma_{p u}[\mathrm{MPa}]$ & $\sigma_{p u} / f_{c p}[-]$ & $\mu[-]$ & $\sigma_{p u}[\mathrm{MPa}]$ & $\sigma_{p u} / f_{c p}[-]$ & $\mu[-]$ \\
\hline 310101 & 30 & 8 & 187.39 & 4.84 & -0.6 & 56.26 & 1.45 & 0.4 \\
\hline 310102 & 25 & 6 & 600.18 & 15.50 & -0.7 & 68.57 & 1.77 & 0.4 \\
\hline 310103 & 25 & 6 & 149.67 & 3.86 & -0.5 & 51.62 & 1.33 & 0.4 \\
\hline 310104 & 25 & 8 & 193.58 & 5.00 & -0.5 & 70.56 & 1.82 & 0.4 \\
\hline 310201 & 25 & 6 & 547.45 & 14.35 & -0.7 & 60.57 & 1.59 & 0.4 \\
\hline 310202 & 20 & 6 & 190.92 & 5.01 & 0.0 & 131.08 & 3.44 & 0.4 \\
\hline 310302 & 30 & 8 & 256.10 & 5.21 & -0.8 & 50.78 & 1.03 & 0.4 \\
\hline 310304 & 25 & 6 & 258.09 & 5.25 & -0.3 & 115.35 & 2.34 & 0.4 \\
\hline
\end{tabular}


Table B.9

Mortar specimens: Obtained values of $\sigma_{p u}$ and $\mu$ when fitted over the post-peak shear displacement range.

\begin{tabular}{|c|c|c|c|c|c|c|c|c|}
\hline \multirow[b]{2}{*}{ \# } & \multirow[b]{2}{*}{$\alpha\left[^{\circ}\right]$} & \multirow[b]{2}{*}{$D_{\text {steel }}[\mathrm{mm}]$} & \multicolumn{3}{|c|}{ Fitted: $\sigma_{p u} \mu$} & \multicolumn{3}{|l|}{ Fitted: $\sigma_{p u}$} \\
\hline & & & $\sigma_{p u}[\mathrm{MPa}]$ & $\sigma_{p u} / f_{c p}[-]$ & $\mu[-]$ & $\sigma_{p u}[\mathrm{MPa}]$ & $\sigma_{p u} / f_{c p}[-]$ & $\mu[-]$ \\
\hline 310401 & 30 & 6 & 189.63 & 5.22 & -0.6 & 31.73 & 0.87 & 0.4 \\
\hline 310402 & 20 & 6 & 437.14 & 12.03 & -0.5 & 82.85 & 2.28 & 0.4 \\
\hline 310404 & 20 & 8 & 288.77 & 7.95 & -0.5 & 73.23 & 2.01 & 0.4 \\
\hline 310405 & 25 & 8 & 264.98 & 7.29 & -0.7 & 42.25 & 1.16 & 0.4 \\
\hline 310505 & 20 & 8 & 379.15 & 8.99 & -0.5 & 96.71 & 2.29 & 0.4 \\
\hline 310506 & 30 & 8 & 307.40 & 7.29 & -0.7 & 45.22 & 1.07 & 0.4 \\
\hline 310406 & 30 & 8 & 258.25 & 7.11 & -0.7 & 36.11 & 0.99 & 0.4 \\
\hline
\end{tabular}

\section{B.2. Two-Phase model enhancement: surface alterations}

Table B.10

Concrete specimens: Obtained values of $\sigma_{p u}$ and $\mu$ for concrete specimens when fitted over the post-peak shear displacement range considering surface alterations.

\begin{tabular}{|c|c|c|c|c|c|c|c|c|}
\hline$\#$ & $\alpha\left[^{\circ}\right]$ & $D_{\text {steel }}[\mathrm{mm}]$ & \multicolumn{3}{|c|}{ Fitted: $\sigma_{p w} \mu$} & \multicolumn{3}{|l|}{ Fitted: $\sigma_{p u}$} \\
\hline 310101 & 30 & 8 & 1049.86 & 27.11 & -1.1 & 62.18 & 1.61 & 0.4 \\
\hline 310103 & 25 & 6 & 532.79 & 13.76 & -0.9 & 57.53 & 1.49 & 0.4 \\
\hline 310104 & 25 & 8 & 721.59 & 18.63 & -1.0 & 76.72 & 1.98 & 0.4 \\
\hline 310201 & 25 & 6 & 2597.30 & 68.10 & -1.0 & 72.22 & 1.89 & 0.4 \\
\hline
\end{tabular}

Table B.11

Mortar specimens: Obtained values of $\sigma_{p u}$ and $\mu$ for concrete specimens when fitted over the post-peak shear displacement range considering surface alterations.

\begin{tabular}{|c|c|c|c|c|c|c|c|c|}
\hline \multirow[b]{2}{*}{ \# } & \multirow[b]{2}{*}{$\alpha\left[^{\circ}\right]$} & \multirow[b]{2}{*}{$D_{\text {steel }}[\mathrm{mm}]$} & \multicolumn{3}{|c|}{ Fitted: $\sigma_{p u}, \mu$} & \multicolumn{3}{|l|}{ Fitted: $\sigma_{p u}$} \\
\hline & & & $\sigma_{p u}[\mathrm{MPa}]$ & $\sigma_{p u} / f_{c p}[-]$ & $\mu[-]$ & $\sigma_{p u}[\mathrm{MPa}]$ & $\sigma_{p u} / f_{c p}[-]$ & $\mu[-]$ \\
\hline 310401 & 30 & 6 & 832.31 & 22.90 & -1.1 & 43.54 & 1.20 & 0.4 \\
\hline 310402 & 20 & 6 & 1655.60 & 45.56 & -0.9 & 97.80 & 2.69 & 0.4 \\
\hline 310404 & 20 & 8 & 1107.12 & 30.46 & -0.9 & 83.50 & 2.30 & 0.4 \\
\hline 310405 & 25 & 8 & 1357.28 & 37.35 & -1.0 & 49.97 & 1.38 & 0.4 \\
\hline 310505 & 20 & 8 & 1428.67 & 33.88 & -0.9 & 110.61 & 2.62 & 0.4 \\
\hline 310506 & 30 & 8 & 1881.46 & 44.61 & -1.2 & 56.15 & 1.33 & 0.4 \\
\hline 310406 & 30 & 8 & 1575.41 & 43.35 & -1.2 & 45.04 & 1.24 & 0.4 \\
\hline
\end{tabular}

\section{References}

[1] A. Muttoni, M. Fernández Ruiz, Shear strength of members without transverse reinforcement as function of critical shear crack width, ACI Struct. J. Am. Concr. Inst. 105 (2) (2008) 163-172 https://infoscience.epfl.ch/record/116126 (ACI Structural Journal).

[2] F. Cavagnis, M. Fernández Ruiz, A. Muttoni, Shear failures in reinforced concrete members without transverse reinforcement: an analysis of the critical shear crack development on the basis of test results, Eng. Struct. 103 (2015) 157-173, https:// doi.org/10.1016/j.engstruct.2015.09.015 (Elsevier).

[3] F. Cavagnis, M. Fernández Ruiz, A. Muttoni, An analysis of the shear-transfer actions in reinforced concrete members without transverse reinforcement based on refined experimental measurements, Struct. Concr. 19(1) (2018) 49-64, https:// doi.org/10.1002/suco.201700145 (Wiley).

[4] R.C. Fenwick, T. Pauley, Mechanisms of shear resistance of concrete beams, J. Struct. Div. Proc. ASCE 94 (10) (1968) 2325-2350 https://cedb.asce.org/ CEDBsearch/record.jsp?dockey $=0015765$ (ASCE)

[5] H.P.J. Taylor, Investigation of the forces carried across cracks in reinforced beams in shear by interlock of aggregate, Cement and Concrete Association, 1970, https:// trid.trb.org/view/21744.

[6] J.C. Walraven, Fundamental analysis of aggregate interlock, ASCE J. Struct. Div. 107 (11) (1981) 2245-2270 https://cedb.asce.org/CEDBsearch/record.jsp? dockey $=0010563$.

[7] B. Li, K. Maekawa, H. Okamura, Contact density model for stress transfer across cracks in concrete, J. Fac. Eng. 40 (1) (1989) 9-52 (The University of Tokyo).

[8] P. Gambarova, C. Karakoç, A new approach to the analysis of the confinement role in regularly cracking concrete elements, Transactions of the 7th International
Conference on Structural Mechanics in Reactor Technology, H 1983, pp. 251-261 https://inis.iaea.org/search/search.aspx?orig_q=RN:15048850.

[9] S. Dei Poli, P. Gambarova, C. Karakoç, Aggregate interlock role in R.C. thin-webbed beams in shear, J. Struct. Eng. 113 (1) (1987) 1-19, https://doi.org/10.1061/ (ASCE)0733-9445(1987)113:1(1).

[10] F.J. Vecchio, M.P. Collins, The modified compression-field theory for reinforced concrete elements subjected to shear, ACI J. 83 (2) (1986) 219-231.

[11] J.S. Jacobsen, Constitutive Mixed Mode Behavior of Cracks in Concrete: Experimental Investigations of Material Modeling, Ph.D. thesis Technical University of Denmark, Kongens Lyngby, Denmark, 2012.

[12] P.M. Calvi, E.C. Bentz, M.P. Collins, Pure mechanics crack model for shear stress transfer in cracked reinforced concrete, ACI Struct. J. (114) (2017) 545-554, https://doi.org/10.14359/51689460.

[13] T. Ulaga, Betonbauteile mit Stab-und Lamellenbewehrung Verbund-und Zuggliedmodellierung ETH Library, Ph.D. thesis ETH, Zurich, Switzerland, 2003, https://doi.org/10.3929/ethz-a-004568673.

[14] R. Guidotti, Poinçonnement des planchers-dalles avec colonnes superposées fortement sollicitées, Ph.D. thesis EPFL, Lausanne, Switzerland, 2010https://infoscience. epfl.ch/record/150264.

[15] A. Muttoni, M. Fernández Ruiz, J.T. Simões, The theoretical principles of the critical shear crack theory for punching shear failures and derivation of consistent closedform design expressions, Struct. Concr. 19 (1) (2018) 174-190, https://doi.org/10 1002/suco.201700088.

[16] W.B. Fuller, S.E. Thompson, The laws of proportioning concrete, Trans. Am. Soc. Civ. Eng. LIX (2) (1907) 67-143.

[17] M. Tirassa, M. Fernández Ruiz, A. Muttoni, Modern experimental research techniques for a consistent understanding of aggregate interlocking, 12th fib International PhD Symposium in Civil Engineering, Czech Technical University in Prague, 
Prague, Czech Republic, 2018, https://infoscience.epfl.ch/record/257222 (29-31 August).

[18] T.R. Thomas, Rough Surfaces, second ed., Imperial College Press, London, 1999, https://doi.org/10.1142/p086.

[19] B.N.J. Persson, O. Albohr, U. Tartaglino, A.I. Volokitin, E. Tosatti, On the nature of surface roughness with application to contact mechanics, sealing, rubber friction and adhesion, J. Phys. Condens. Matter 17 (1) (2005) 1-62, https://doi.org/10. 1088/0953-8984/17/1/R01 (IOP Publishing).

[20] B. Bujadham, K. Maekawa, Qualitative studies on mechanisms of stress transfer across cracks in, Proc. JSCE 17 (451) (1992) 265-275, https://doi.org/10.2208/ jscej.1992.451.

[21] CEN European Committee for Standardization, EN 196-1. Methods of Testing Cement - Part 1: Determination of Strength, CEN, 1994.

[22] VSS (Swiss Association of Road and Transportation Experts), SN670115 Gesteinskörnungen: Qualitative und quantitative, Mineralogie und Petrographie, Zurich, Switzerland, 2005.

[23] fib (International Federation for Structural Concrete), fib Model Code for Concrete Structures 2010, Ernst \& Sohn. doi: 10.1002/9783433604090.

[24] A. Muttoni, Die Anwendbarkeit der Plastizitätstheorie in der Bemessung von Stahlbeton, Ph.D. thesis ETH, Zurich, Switzerland, 1989, https://doi.org/10.1007/ 978-3-0348-5598-3.

[25] M. Fernández Ruiz, A. Muttoni, On development of suitable stress fields for structural concrete, ACI Struct. J. Am. Concr. Inst. 104 (4) (2007) 495-502, https:// doi.org/10.14359/18780.

[26] K.L. Johnson, Contact Mechanics, Cambridge University Press, Cambridge, 1985, https://doi.org/10.1017/CBO9781139171731.

[27] R. Hill, The Mathematical Theory of Plasticity, Oxford University Press, Oxford, 1950.

[28] F.E. Richart, A. Brandtzaeg, R.L. Brown, A Study of the Failure of Concrete under Combined Compressive Stresses, Engineering Experiment Station Bulletin 185, University of Illinois, 1928, http://hdl.handle.net/2142/4277.

[29] M.P. Nielsen, L.C. Hoang, Limit Analysis and Concrete Plasticity, third edition, CRC Press, Boca Raton, Florida, 2010.

[30] I. Kalliomäki, A. Vehtari, J. Lampinen, Shape analysis of concrete aggregates for statistical quality modeling, Mach. Vis. Appl. 16 (3) (2005) 197-201, https://doi. org/10.1007/s00138-004-0172-3 (Springer).

[31] P. Wriggers, S.O. Moftah, Mesoscale models for concrete: homogenisation and damage behaviour, Finite Elem. Anal. Des. 42 (7) (2006) 623-636, https://doi.org/ 10.1016/j.finel.2005.11.008 (Elsevier).

[32] P.R. Nayak, Random process model of rough surfaces, J. Lubr. Technol. 93 (3) (1971) 398-407, https://doi.org/10.1115/1.3451608.

[33] V.A. Yastrebov, G. Anciaux, J.F. Molinari, From infinitesimal to full contact between rough surfaces: evolution of the contact area, Int. J. Solids Struct. 52 (2015) 83-102, https://doi.org/10.1016/j.ijsolstr.2014.09.019 (Elsevier). 